\title{
HOMOTOPY THEORY OF STRATIFIED SPACES
}

\author{
SYLVAIN DOUTEAU
}

\begin{abstract}
In this article, we construct a cofibrantly generated model structure on the category of spaces stratified over a fixed poset, and show that it is Quillen-equivalent to a category of diagrams of simplicial sets. Then, considering all those model structures together, we construct a cofibrantly generated model structure on the category of all stratified spaces. In both model categories, weak-equivalences are characterized by stratified homotopy groups.
\end{abstract}

\section{Contents}

Introduction 1

Linear overview 3

1. Strongly stratified spaces and stratified homotopy groups 3

1.1. Strongly stratified, strongly filtered spaces and filtered simplicial sets 3

1.2. Stratified homotopy groups 6

1.3. Stratified and filtered spaces 8

2. The model category of filtered spaces 9

2.1. The category of diagrams 9

2.2. The model category $\operatorname{Top}_{N(P)}$

2.3. Quillen equivalence between $\operatorname{Top}_{N(P)}$ and $\operatorname{Diag}_{P}$

2.4. Quillen equivalence between the categories of filtered and strongly filtered spaces 14

3. A model category for stratified spaces 17

3.1. Comparing model structures between posets 17

3.2. The model category Strat 19

Appendix A. A property of some colimits over particular posets 22

References 25

\section{INTRODUCTION}

Stratified spaces are omnipresent in geometry and topology whenever dealing with singular objects. Stratifications were first defined by H. Whitney Whi65 for algebraic varieties, and R. Thom Tho69 for analytic varieties. Both defined a stratification as a decomposition of a variety $X$ into smooth pieces called strata, $X=\coprod_{p \in P} X_{p}$, together with some gluing conditions. The study of stratified spaces developed through the generalization of topological invariants of manifolds to the stratified setting. Among those invariants, intersection cohomology - introduced in 1980 by M. Goresky and R. MacPherson in GM80 and GM83 - is one of the first, and probably the most well-known. It is a "cohomology theory" suited for the study of stratified spaces and for which 
pseudo-manifolds - such as those defined by R. Thom and H. Whitney - satisfy Poincaré duality. Since then, numerous other invariants have been defined for stratifed spaces, such as

- The category of exit-paths, and its higher-categorical generalizations, first defined by MacPherson (unpublished) and studied by Treuman Tre09, J. Woolf Woo09] and J. Lurie [ur.

- Singular chains and cochains complexes carrying cap and cup products and computing intersection (co)homology have been defined by H.King Kin85 and G. Friedman and J. McClure [FM13, Fri].

- A rational homotopy theory has been developed by D. Chataur, M. Saralegui and D. Tanré CSAT18.

- Generalized intersection homology theories have been proposed by M. Banagl [Ban10].

All those invariants are compatible with strata-preserving homotopy equivalences, though they are not preserved by regular homotopy equivalences. This begs the question - already asked by Goresky and MacPherson about intersection cohomology $\left[\mathrm{B}^{+} 08\right.$, Problem \#4]. What is the corresponding homotopy category of stratified spaces that factor those invariants?

Several partial answers have been given toward the resolution of this problem. D. Miller Mil13, gave a characterization of strata-preserving homotopy equivalences for the class of homotopically stratified spaces defined by Quinn Qui88. Later, in his thesis, S. Nand-Lal NL19 defined a partial model structure on the subcategory of "fibrant stratified spaces" using an adjunction with the category of simplicial sets. In another direction, both P.Haine Hai18 and the author Dou18 independently defined model structures on a category of filtered simplicial sets. All those results allowed to further the understanding of the homotopy theory of stratified spaces, but a description of a model structure on the entire category of stratified spaces was still missing.

The goal of this paper is to construct and characterize such a model structure. As is classical, we define a stratified space as a topological space $X$ together with a continuous map to a partially ordered set of strata $\varphi_{X}: X \rightarrow P$ (see for example [Lur, Definition A.5.1]). We use the stratified homotopy groups, a stratified homotopy invariant introduced in Dou18, to characterize stratified weak-equivalences and prove the following (Theorem 3.6).

Theorem 1. The category of stratified spaces admits a cofibrantly generated model structure in which a map $f:(X \rightarrow P) \rightarrow(Y \rightarrow Q)$ is a weak-equivalence if and only if it induces isomorphisms on all stratified homotopy groups.

$$
s \pi_{n}(f): s \pi_{n}((X \rightarrow P), \phi) \rightarrow s \pi_{n}((Y \rightarrow Q), f \circ \phi) \circ \widehat{f}
$$

In order to prove Theorem 1, we first work over a fixed poset $P$, and construct a model structure on the category of spaces filtered over $P$, $\operatorname{Top}_{P}$ (Theorem 2.15).

Theorem 2. There exists a cofibrantly generated model structure on Top $p_{P}$ in which a map $f:(X \rightarrow$ $P) \rightarrow(Y \rightarrow P)$ is a weak-equivalence if and only if it induces isomorphisms on all stratified homotopy groups.

$$
s \pi_{n}(f): s \pi_{n}((X \rightarrow P), \phi) \rightarrow s \pi_{n}((Y \rightarrow P), f \circ \phi)
$$

To construct this model structure, we consider an adjunction with a category of diagrams of simplicial sets

$$
\operatorname{Top}_{P} \leftrightarrow \operatorname{Fun}\left(R(P)^{o p}, \text { sSet }\right)
$$

where $R(P)$ can be succintly described as the subdivision of $P$. The projective model structure on $\operatorname{Fun}\left(R(P)^{o p}\right.$, sSet) can then be transported onto $\operatorname{Top}_{P}$ along the adjunction (1) by HKRS17 Corollary 3.3.4]. We then have the following characterization of $\operatorname{Top}_{P}$ (Theorems [2.15] and 2.12). 
Theorem 3. The adjunction (1) is a Quillen-equivalence.

Using this Quillen-Equivalence, P. Haine Hai18 was able to relate the aforementioned $\infty$ category of filtered spaces, Top ${ }_{P}$, and the $\infty$-category of décollages, Déc $P$ considered by C. Barwick, S. Glassman and himself in BGH18.

In order to prove Theorem [3, we introduce a more rigid notion of stratification. A strongly stratified space will be a space $X$ together with a strong stratification $\varphi_{X}: X \rightarrow\|N(P)\|$. This allows one to consider the preimages of any point $t \in\|N(P)\|, \varphi_{X}^{-1}(t) \subset X$, in addition to the strata of $X$. This idea was first considered by A. Henriques Hen - for the study of filtered simplicial sets through their realization - and proved to be extremely useful for the study of strongly stratified spaces.

Linear overview. Section 1 covers the definitions needed in the rest of the paper. In section 1.1. we introduce strongly stratified spaces, strongly stratified map, and give some examples. The section also contains the notions of stratified homotopy equivalences and of various useful functors. In section 1.2, we recall the definition of stratified homotopy groups from [Dou18, although the definition is given in terms of strongly stratified spaces. In section 1.3, we make explicit the relationship between stratified and strongly stratified spaces.

Section 2 contains the proof of Theorems 2 and 3 . It is concerned with the homotopy theory of spaces (strongly) filtered over a fixed poset $P$. In section 2.1. we recall the projective model structure on a category of diagrams. The model structure on the category of strongly filtered spaces, $\operatorname{Top}_{N(P)}$ is constructed in section 2.2 (Theorem 2.8), and its Quillen-equivalences with the category of diagrams $\operatorname{Diag}_{P}$ (Theorem 2.12) and the category of filtered spaces Top $_{P}$ (Theorem 2.15] are proved in section [2.3] and 2.4 respectively.

Section 3 contains the proof of Theorem 1. In section 3.1, we prove that any map of posets $\alpha: P \rightarrow Q$ induces Quillen-adjunctions between $\operatorname{Top}_{P}$ and $\operatorname{Top}_{Q}$ which is a Quillen-equivalence if and only if $\alpha$ is an isomorphism. This is precisely hypothesis $(Q)$ in [CM17, Theorem 4.2], which we apply in section 3.2 to produce a model structure on Strat (Theorem 3.6).

Appendix $\mathrm{A}$ contains the proof of a simple but technical fact about particular colimits appearing in the proof of Theorem 2.12 .

\section{Strongly StRATified SPACES AND StRATified Homotopy GRoups}

In this section, we introduce the category of strongly stratified spaces, StrStrat, and provide a few examples. We then recall a complete definition of the stratified homotopy groups from [Dou18] expressed in terms of strongly stratified spaces. Finally, after recalling the classical definition of a stratified space, we explain the relation between the categories of stratified spaces and of strongly stratified spaces.

1.1. Strongly stratified, strongly filtered spaces and filtered simplicial sets. Throughout this paper, Top stands for the category of $\Delta$-generated spaces in the sense of [Dug03. In particular, all topological spaces are assumed to be $\Delta$-generated. This is needed in order for the category Top (and in turn the categories $\operatorname{Top}_{P}$ and $\operatorname{Top}_{N(P)}$ ) to be a locally presentable category.

Definition 1.1. A strongly stratified space is the data of a triple $\left(X, P, \varphi_{X}\right)$ where

- $X$ is a topological space,

- $P$ is a partially ordered set,

- $\varphi_{X}: X \rightarrow\|N(P)\|$ is a continuous map, where $\|N(P)\|$ is the realisation of the nerve of $P$. 
A (strongly) stratified map between strongly stratified spaces $f:\left(X, P, \varphi_{X}\right) \rightarrow\left(Y, Q, \varphi_{Y}\right)$ is the data of

- a continuous map $f: X \rightarrow Y$,

- a map of posets $\widehat{f}: P \rightarrow Q$,

such that the following diagram commutes

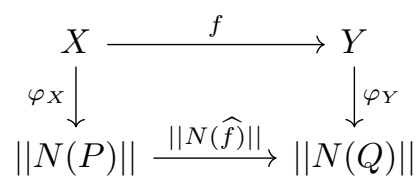

The category StrStrat is the category of strongly stratified spaces and (strongly) stratified maps.

Remark 1.2. We will drop the adverb "strongly" in the expression "strongly stratified map", since all the map we will be considering between strongly stratified spaces will be strongly stratified.

Example 1.3. - The most immediate class of example of strongly stratified spaces comes from triangulated spaces. Let $X \simeq\|K\|$ be a triangulated space, and assume that some decomposition of $X$ into sub-triangulated spaces - the strata of $\mathrm{X}$ - is given $X=\bigsqcup_{p \in P} X_{p}$, with $X_{p}=\left\|K_{p}\right\|$, and $K_{p} \subset K$. This gives a map between set $\varphi_{K}: K_{0} \rightarrow P$ sending a vertex $v \in K$ to the $p \in P$ such that $v \in K_{p}$. If the strata of $X$ satisfy the frontier condition (we refer the reader to TT18, Section 3.1] for a definition of the frontier condition and a discussion of the various possible assumptions on a stratification), one can define a partial order on the set of strata of $X$ as

$$
p \leq q \Leftrightarrow X_{p} \cap \bar{X}_{q} \neq \emptyset .
$$

The map of sets $\varphi_{K}$ then extends to a map of simplicial sets

$$
\varphi_{K}: K \rightarrow N(P)
$$

Taking the realization of this map, one then has a strong stratification on $X$

$$
\varphi_{X}: X \simeq\|K\| \rightarrow\|N(P)\|
$$

satisfying $\varphi_{X}^{-1}(\{p\})=X_{p}$ for all $p \in P$. See Definitions 1.7 and 1.8,

- Let $L$ be some topological space, and define its (closed) cone as

$$
\bar{c}(L)=L \times[0,1] / L \times\{0\} .
$$

Identifying $\left\|N\left(\left\{p_{0}<p_{1}\right\}\right)\right\|$ with $[0,1]$ gives a strong stratification on $\bar{c}(L)$ :

$$
\varphi_{\bar{c}(L)}=\operatorname{pr}_{[0,1]}: \bar{c}(L) \simeq L \times[0,1] / L \times\{0\} \rightarrow[0,1] \simeq\left\|N\left(\left\{p_{0}<p_{1}\right\}\right)\right\| .
$$

- More generally, let $X$ be a pseudo-manifold with isolated singularities. That is, $X$ is a topological manifold outside of a discrete subset of points, and those points have neighborhoods homeomorphic to (closed) cones. Let $\left\{x_{i}\right\}_{i \in I} \subset X$ be the set of singular points, and fix some mutually disjoint neighborhood of the $x_{i}, F_{i} \subset X$, such that $F_{i} \simeq \bar{c}\left(L_{i}\right)$ for some manifold $L_{i}$, for $i \in I$. Then, each of the $F_{i}$ admits a strong stratification to $\left\|N\left(\left\{p_{i}<q\right\}\right)\right\|$. Consider the poset $P=\left\{p_{i} \mid i \in I\right\} \cup\{q\}$, with relations $p_{i}<q$ for all $i \in I$. Taking all the strong stratifications $\varphi_{\bar{c}\left(L_{i}\right)}$ together - and defining $\varphi_{X}$ on the complement of the $F_{i}$ as $q$ gives a strong stratification on $X: \varphi_{X}: X \rightarrow\|N(P)\|$. 
- Let $\varphi_{L}: L \rightarrow\|N(P)\|$ be a strongly stratified space. Define the poset $c(P)$ as $P \amalg\{-\infty\}$, with $-\infty<p$ for all $p \in P$. Notice that $\|N(c(P))\|$ can be identified with $c(\|N(P)\|)$. With this identification, $\bar{c}(L)$ admits a strong stratification to $\|N(c(P))\|$.

$$
\varphi_{\bar{c}(L)}=\bar{c}\left(\varphi_{L}\right): \bar{c}(L) \rightarrow \bar{c}\|N(P)\| \simeq\|N(c(P))\| .
$$

- Taking both previous examples together, one can define recursively a strong stratification on any pseudo-manifold given compatible trivializing neighborhoods for all strata.

Definition 1.4. Let $f, g:\left(X, P, \varphi_{X}\right) \rightarrow\left(Y, Q, \varphi_{Y}\right)$ be two stratified maps. We say that $f$ and $g$ are stratified homotopic if there exists a stratified homotopy $H:\left(X \times[0,1], P, \varphi_{X} \circ p r_{X}\right) \rightarrow\left(Y, Q, \varphi_{Y}\right)$ such that $H_{\mid X \times\{0\}}=f$ and $H_{\mid X \times\{1\}}=g$. A stratified map $f:\left(X, P, \varphi_{X}\right) \rightarrow\left(Y, Q, \varphi_{Y}\right)$ is a stratified homotopy equivalence if there exists a stratified map $g:\left(Y, Q, \varphi_{Y}\right) \rightarrow\left(X, P, \varphi_{X}\right)$ such that $f \circ g$ and $g \circ f$ are stratified homotopic to $I d_{Y}$ and $I d_{X}$ respectively.

Remark 1.5. From the definition of a stratified homotopy equivalence, we deduce that if $\left(X, P, \varphi_{X}\right)$ and $\left(Y, Q, \varphi_{Y}\right)$ are stratified homotopy equivalent, then $P$ and $Q$ must be isomorphic as posets. In particular, in order to understand the homotopy theory of stratified spaces, it is enough to work over a fixed poset. This is what we will call the filtered setting. This leads us to the following definition.

Definition 1.6. Let $P$ be a partially ordered set, the category $\operatorname{Top}_{N(P)}$ of strongly filtered spaces over $P$ is the subcategory of StrStrat whose objects are the strongly stratified spaces $\left(X, P, \varphi_{X}\right)$, and whose maps $f:\left(X, P, \varphi_{X}\right) \rightarrow\left(Y, P, \varphi_{Y}\right)$ satisfy $\widehat{f}=I d_{P}$. When working with filtered objects, if the poset $P$ is clear from the context, we will drop it from the notation and write $\left(X, \varphi_{X}\right)$ instead of $\left(X, P, \varphi_{X}\right)$.

Definition 1.7. Let $P$ be a partially ordered set. A filtered simplicial set over $P$ is the data of a pair $\left(K, \varphi_{K}\right)$, where

- $K$ is a simplicial set,

- $\varphi_{K}: K \rightarrow N(P)$ is a simplicial map, where $N(P)$ is the nerve of $P$.

A filtered map between filtered simplicial sets, $f:\left(K, \varphi_{K}\right) \rightarrow\left(L, \varphi_{L}\right)$ is a simplicial map $f: K \rightarrow L$ such that the following diagram commutes

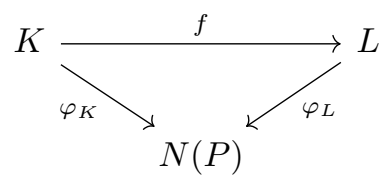

The category $\operatorname{sSet}_{P}$ is the category of filtered simplicial sets over $P$ and filtered maps.

Definition 1.8. We define the strongly filtered realization functor $\|-\| \|_{N(P)}: \operatorname{sSet}_{P} \rightarrow \operatorname{Top}_{N(P)}$ as follows. On objects, $\left\|\left(K, \varphi_{K}: K \rightarrow N(P)\right)\right\|_{N(P)}=\left(\|K\|,\left\|\varphi_{K}\right\|\right)$, and on maps, $\| f:\left(K, \varphi_{K}\right) \rightarrow$ $\left(L, \varphi_{L}\right)\left\|_{N(P)}=\right\| f\|:\|\left(K, \varphi_{K}\right)\left\|_{N(P)} \rightarrow\right\|\left(L, \varphi_{L}\right) \|_{N(P)}$.

Proposition 1.9. The functor $\|-\|_{N(P)}: \operatorname{sSet}_{P} \rightarrow \operatorname{Top}_{N(P)}$ admits a right adjoint, $\operatorname{Sing}_{N(P)}: \operatorname{Top}_{N(P)} \rightarrow$ $\operatorname{sSet}_{P}$ defined as the following pullback

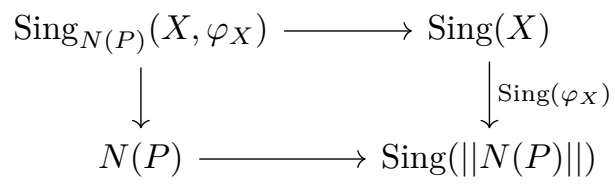


Proof. See for example [Lur, Definition A.6.2]

\subsection{Stratified homotopy groups.}

Definition 1.10. Let $P$ be a poset. We define $R(P)$ as the full subcategory of $\operatorname{sSet}_{P}$ whose objects are the non degenerate simplices of $N(P), \varphi: \Delta^{n} \rightarrow N(P)$. We will write $\Delta^{\varphi}$, or $\left\{p_{0}<\cdots<p_{n}\right\}$ for such a simplex, where $p_{i}$ is the image by $\varphi$ of the $i$-th vertex of $\Delta^{n}$. The category $\operatorname{Diag}_{P}$ of simplicial diagrams over $P$ is the category of functors

$$
\operatorname{Diag}_{P}=\operatorname{Fun}\left(R(P)^{o p}, \text { sSet }\right)
$$

Definition 1.11. Let $\left(X, \varphi_{X}\right)$ and $\left(Y, \varphi_{Y}\right)$ be two strongly filtered spaces. The inclusion

$$
\operatorname{Hom}_{\operatorname{Top}_{N(P)}}\left(\left(X, \varphi_{X}\right),\left(Y, \varphi_{Y}\right)\right) \subset \mathcal{C}^{0}(X, Y),
$$

induces a topology on $\operatorname{Hom}_{\operatorname{Top}_{N(P)}}\left(\left(X, \varphi_{X}\right),\left(Y, \varphi_{Y}\right)\right)$. Write $\mathcal{C}_{P}^{0}\left(\left(X, \varphi_{X}\right),\left(Y, \varphi_{Y}\right)\right)$ for the corresponding topological space. This defines a functor

$$
\mathcal{C}_{N(P)}^{0}: \operatorname{Top}_{N(P)}^{o p} \times \operatorname{Top}_{N(P)} \rightarrow \text { Top }
$$

Definition 1.12. We define the functor $D: \operatorname{Top}_{N(P)} \rightarrow \operatorname{Diag}_{P}$ as follows

$$
\begin{aligned}
\operatorname{Top}_{N(P)} & \rightarrow \operatorname{Diag}_{P} \\
\left(X, \varphi_{X}\right) & \mapsto \operatorname{Sing}\left(\mathcal{C}_{N(P)}^{0}\left(\|-\|_{N(P)},\left(X, \varphi_{X}\right)\right)\right)
\end{aligned}
$$

Definition 1.13. Let $\left(X, \varphi_{X}\right)$ be a strongly filtered space over $P$. The stratified set of connected components of $\left(X, \varphi_{X}\right)$ is the functor $s \pi_{0}\left(X, \varphi_{X}\right): R(P)^{o p} \rightarrow$ Set, obtained by composing $D$ with the functor $\pi_{0}$ : sSet $\rightarrow$ Set.

We want to define the stratified homotopy groups of a strongly stratified space $\left(X, P, \varphi_{X}\right)$, as the collection of homotopy groups of $D\left(\left(X, \varphi_{X}\right)\right)\left(\Delta^{\varphi}\right)$ for all $\Delta^{\varphi}$. In order to do so, one should first point each simplicial set $D\left(\left(X, \varphi_{X}\right)\right)\left(\Delta^{\varphi}\right)$ in a way that is compatible with the maps $D\left(\left(X, \varphi_{X}\right)\right)\left(\Delta^{\varphi}\right) \rightarrow$ $D\left(\left(X, \varphi_{X}\right)\right)\left(\Delta^{\psi}\right)$ induced by the inclusions $\Delta^{\psi} \rightarrow \Delta^{\varphi}$. One way of obtaining such a collection of pointings is to consider a filtered map $\|N(P)\|_{N(P)} \rightarrow\left(X, \varphi_{X}\right)$. However, such a map need not always exist, even for non-pathological spaces, as illustrated by Example 1.14 Instead, we will consider partial collection of pointings, induced by strongly filtered maps $\left\|\Delta^{\varphi}\right\|_{N(P)} \rightarrow\left(X, \varphi_{X}\right)$ (Definition 1.15).

Example 1.14 ([NL19, Example 10.1.0.10]). Consider the stratified space of figure 1, obtained as the realization of some stratified simplicial set. It is stratified over $P=\left\{a_{1}, a_{2}, a_{3}, b_{1}, b_{2}, b_{3}, c\right\}$ with relations $a_{i}<b_{i-1}, b_{i+1}, 1 \leq i \leq 3$ where $i+1$ and $i-1$ are to be understood mod 3 , and $b_{j}<c$ for $1 \leq j \leq 3$. One checks that $s \pi_{0}\left(X, P, \varphi_{X}\right)$ is given by the functor

$$
\begin{aligned}
s \pi_{0}\left(X, P, \varphi_{X}\right): R(P)^{o p} & \rightarrow \text { Set } \\
\Delta^{\varphi} & \mapsto\{*\}
\end{aligned}
$$

And so, $\left(X, P, \varphi_{X}\right)$ is "connected" as a stratified space in the strongest possible sense, and it is the realization of a stratified simplicial set. Yet, there exists no stratified "pointing" of the form $\phi:\|N(P)\| \rightarrow\left(X, P, \varphi_{X}\right)$.

Definition 1.15. Let $\left(X, \varphi_{X}\right)$ be a space strongly filtered over $P$. A filtered pointing of $\left(X, \varphi_{X}\right)$ is the data of a non-degenerate simplex of $N(P), \Delta^{\varphi}$ and of a strongly filtered map

$$
\phi:\left\|\Delta^{\varphi}\right\|_{N(P)} \rightarrow\left(X, \varphi_{X}\right) .
$$




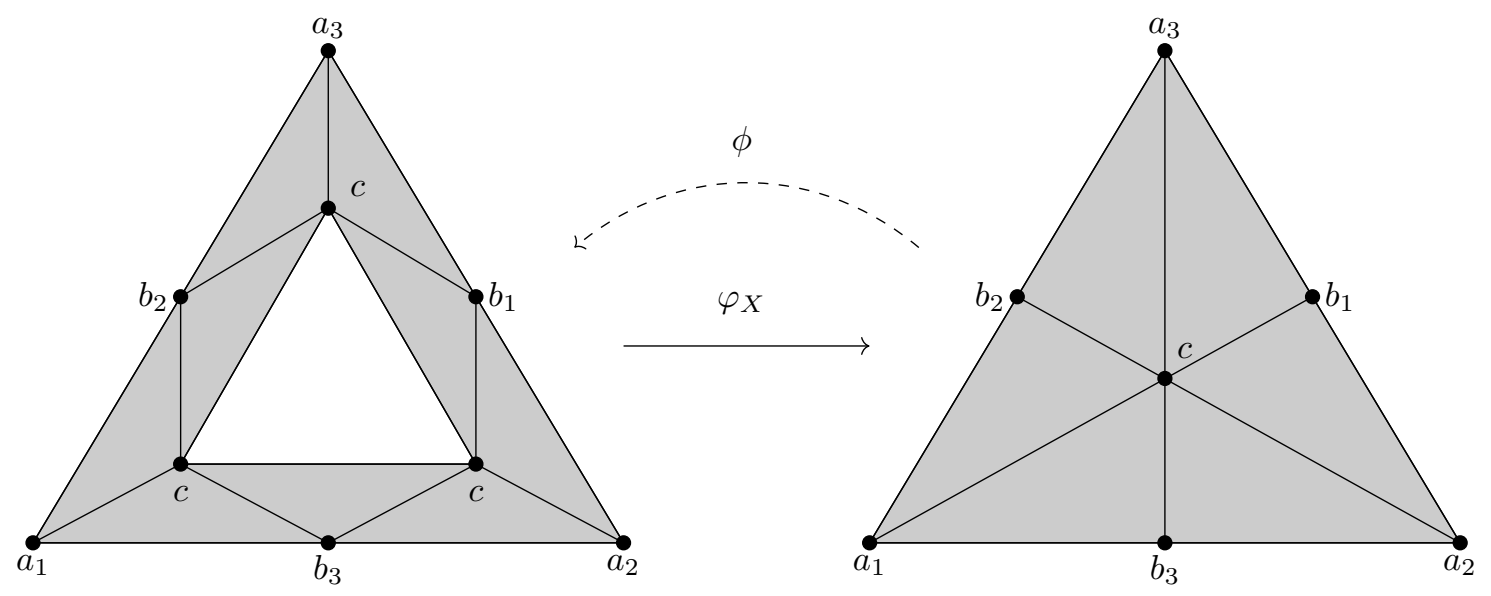

FIgUre 1 . The space $X$ with a stratification over $\|N(P)\|$, with $P=\left\{a_{1}, a_{2}, a_{3}, b_{1}, b_{2}, b_{3}, c\right\}$

Remark 1.16. If $\varphi: \Delta^{n} \rightarrow N(P)$ is a non-degenerate simplex of $N(P)$, the vertices of $\Delta^{n}$ are sent by $\varphi$ to an increasing sequence of elements of $P, \varphi\left(e_{0}\right)<\varphi\left(e_{1}\right)<\cdots<\varphi\left(e_{n}\right)$. We will write $\operatorname{Im}(\varphi)$ for this totally ordered subset of $P$. Note that $R(\operatorname{Im}(\varphi))$ is canonically equivalent to the full subcategory of $R(P)$ whose objects are the $\Delta^{\psi} \subseteq \Delta^{\varphi}$.

Definition 1.17. Let $\left(X, \varphi_{X}\right)$ be a space strongly filtered over $P$, and let $\phi:\left\|\Delta^{\varphi}\right\|_{N(P)} \rightarrow\left(X, \varphi_{X}\right)$ be a filtered pointing of $\left(X, \varphi_{X}\right)$. We define the pointed diagram of $\left(X, \varphi_{X}\right)$,

$$
D_{\phi}\left(X, \varphi_{X}\right): R(\operatorname{Im}(\varphi))^{o p} \rightarrow \operatorname{sSet}_{*}
$$

as the restriction of the functor $D\left(\left(X, \varphi_{X}\right)\right)$ to the full subcategory $R(\operatorname{Im}(\varphi))^{o p} \subseteq R(P)^{o p}$, where each $D\left(X, \varphi_{X}\right)\left(\Delta^{\psi}\right)$ is pointed by $\phi_{\left\|\Delta^{\psi}\right\|_{N(P)}}$ for all $\Delta^{\psi} \subseteq \Delta^{\varphi}$. Here, sSet $_{*}$ is the category of pointed simplicial sets. Let $k \geq 1$, the $k$-th stratified homotopy group of $\left(X, \varphi_{X}\right)$ with respect to the pointing $\phi$ is the functor with values in the category of groups

$$
s \pi_{k}\left(\left(X, \varphi_{X}\right), \phi\right): R(\operatorname{Im}(\varphi))^{o p} \rightarrow \operatorname{Grp},
$$

obtained by composing $D_{\phi}\left(X, \varphi_{X}\right)$ with the $k$-th homotopy group functor $\pi_{k}$ : sSet $_{*} \rightarrow$ Grp.

Remark 1.18. If $P=\{*\}$ is the poset with only one element, then any space $X$ is stratified over $P$. In this case, a stratified pointing is just a point $x \in X$, and the category $R(P)$ has a single object $\Delta^{*}$. For $n \geq 1$, one has $s \pi_{n}(X, x)\left(\Delta^{*}\right)=\pi_{n}(X, x)$ and $s \pi_{0}(X)\left(\Delta^{*}\right)=\pi_{0}(X)$.

The following propositions follow easily from the functoriality of previous definitions.

Proposition 1.19. Let $f:\left(X, \varphi_{X}\right) \rightarrow\left(Y, \varphi_{Y}\right)$ be a filtered map. Then, for all $n \geq 0$ and all stratified pointings of $X, \phi:\left\|\Delta^{\varphi}\right\|_{N(P)} \rightarrow\left(X, \varphi_{X}\right), f$ induces a natural transformation

$$
s \pi_{n}(f): s \pi_{n}\left(\left(X, \varphi_{X}\right), \phi\right) \rightarrow s \pi_{n}\left(\left(Y, \varphi_{Y}\right), f \circ \phi\right)
$$

Proposition 1.20. Let $\left(X, \varphi_{X}\right)$ be a space strongly filtered over $P$ and let $\phi, \phi^{\prime}:\left\|\Delta^{\varphi}\right\|_{N(P)} \rightarrow$ $\left(X, \varphi_{X}\right)$ be two pointings of $\left(X, \varphi_{X}\right)$. Then if $H:\left\|\Delta^{\varphi}\right\|_{N(P)} \times[0,1] \rightarrow\left(X, \varphi_{X}\right)$ is a stratified homotopy between $\phi$ and $\phi^{\prime}$, then $H$ induces isomorphisms for all $n \geq 1$

$$
s \pi_{n}(H): s \pi_{n}\left(\left(X, \varphi_{X}\right), \phi\right) \stackrel{\simeq}{\rightarrow} s \pi_{n}\left(\left(X, \varphi_{X}\right), \phi^{\prime}\right) .
$$


Furthermore, let $f, g:\left(X, \varphi_{X}\right) \rightarrow\left(Y, \varphi_{Y}\right)$ be two filtered maps, if $H^{\prime}:\left(X, \varphi_{X}\right) \times[0,1] \rightarrow\left(Y, \varphi_{Y}\right)$ is a filtered homotopy between $f$ and $g$, then there is a commutative diagram

$$
\begin{array}{r}
s \pi_{n}\left(\left(X, \varphi_{X}\right), \phi\right) \stackrel{s \pi_{n}(f)}{\longrightarrow} s \pi_{n}\left(\left(Y, \varphi_{Y}\right), f \circ \phi\right) \\
\underset{s \pi_{n}(g)}{\simeq} \underset{s \pi_{n}\left(H^{\prime} \circ \phi\right)}{ } \\
s \pi_{n}(Y, g \circ \phi)
\end{array}
$$

Remark 1.21. Although pointings of the form $\phi:\|N(P)\|_{N(P)} \rightarrow\left(X, \varphi_{X}\right)$ need not always exist (see Example 1.14), one might still want to consider them when they do. Given such a pointing, one can still define the stratified homotopy groups of $\left(X, \varphi_{X}\right)$ with respect to $\phi$, and in that case, one gets a functor from the full category $R(P)^{o p}$ to Grp. Furthermore, this functor is still invariant under stratified homotopy in the sense of Propositions 1.19 and 1.20

\subsection{Stratified and filtered spaces.}

Definition 1.22. A stratified space is the data of a triple $\left(X, P, \varphi_{X}\right)$ where

- $X$ is a topological space,

- $P$ is a partially ordered set,

- $\varphi_{X}: X \rightarrow P$ is a continuous map, where $P$ is given the Alexandrov topology.

A stratified map between stratified spaces $f:\left(X, P, \varphi_{X}\right) \rightarrow\left(Y, Q, \varphi_{Y}\right)$ is the data of

- a continuous map $f: X \rightarrow Y$,

- a map of posets $\widehat{f}: P \rightarrow Q$,

such that the following diagram commutes

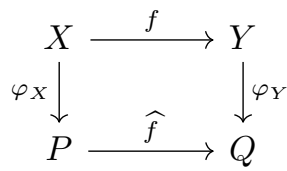

The category Strat is the category of stratified spaces and stratified maps. Let $P$ be some fixed poset, the category of filtered spaces over $P$, Top $P$ is the subcategory of Strat whose objects are of the form $\left(X, P, \varphi_{X}\right)$ and whose maps $f:\left(X, P, \varphi_{X}\right) \rightarrow\left(Y, P, \varphi_{Y}\right)$ verify $\widehat{f}=I d_{P}$.

Recall that a point in $\|N(P)\|$ is represented by a pair, $\left(\left\{p_{0}<\cdots<p_{n}\right\}, t\right)$, where $\left\{p_{0}, \ldots, p_{n}\right\}$ is a simplex of $N(P)$, and $t \in \Delta^{n}$ is a point in the standard $n$-simplex. If one adds the condition that $t$ lies in the interior of $\Delta^{n}$, then this representation is unique.

Definition 1.23. Let $\varphi_{P}:\|N(P)\| \rightarrow P$ be the following morphism

$$
\begin{aligned}
\varphi_{P}:\|N(P)\| & \rightarrow P \\
\left(\left\{p_{0}<\cdots<p_{n}\right\}, t \in \operatorname{Int}\left(\Delta^{n}\right)\right) & \mapsto p_{n}
\end{aligned}
$$

We will write $\varphi_{P} \circ-: \operatorname{Top}_{N(P)} \rightarrow \operatorname{Top}_{P}$ for the functor sending $\left(X, P, \varphi_{X}\right) \in \operatorname{Top}_{N(P)}$ to $\left(X, P, \varphi_{P} \circ\right.$ $\left.\varphi_{X}\right)$. Let $\left(Y, P, \varphi_{Y}\right) \in \operatorname{Top}_{P}$ be a $P$-filtered space. Consider the following pullback

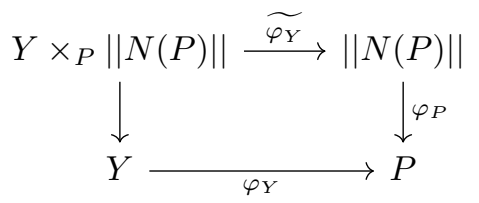


Define the functor $-\times_{P}\|N(P)\|: \operatorname{Top}_{P} \rightarrow \operatorname{Top}_{N(P)}$ as the following functor

$$
\begin{aligned}
-\times_{P}\|N(P)\|: \operatorname{Top}_{P} & \rightarrow \operatorname{Top}_{N(P)} \\
\left(Y, P, \varphi_{Y}\right) & \mapsto\left(Y \times_{P}\|N(P)\|, P, \widetilde{\varphi_{Y}}\right)
\end{aligned}
$$

Proposition 1.24. The functor $-\times_{P}\|N(P)\|$ is right adjoint to the functor $\varphi_{P} \circ-$

Remark 1.25. Thanks to the previous adjonction, one can define an adjunction between the category of filtered simplicial set and of filtered spaces as follows

$$
\|-\|_{P}=\left(\varphi_{P} \circ-\right)\|-\|_{N(P)}: \operatorname{sSet}_{P} \leftrightarrow \operatorname{Top}_{P}: \operatorname{Sing}_{N(P)}\left(-\times_{P}\|N(P)\|\right)=\operatorname{Sing}_{P}
$$

Similarly, one defines the stratified homotopy groups of a filtered space $\left(Y, P, \varphi_{Y}\right)$ as the filtered homotopy groups of $\left(Y \times{ }_{P}\|N(P)\|, P, \widetilde{\varphi_{Y}}\right)$. One checks that this definition coincides with the definition given in Dou18

\section{THE MODEL CATEGORY OF FILTERED SPACES}

We saw in Remark 1.5 that it was sufficient to work over a fixed poset to understand the homotopy theory of stratified spaces. In this section, some poset $P$ is fixed, and we construct and characterize model structures for the categories of filtered and strongly filtered spaces over $P$ - the construction of the model structure on Strat is the content of section 3. We first recall the projective model structure on the category of diagrams of simplicial sets, $\operatorname{Diag}_{P}$ (see Definition 1.10) and then transport this model structure on $\operatorname{Top}_{N(P)}$ (Theorem 2.8). We then go on to show that the two model structure are Quillen-equivalent (Theorem 2.12), and that one can also transport a model structure to $\operatorname{Top}_{P}$ that is Quillen-equivalent to the one on $\operatorname{Top}_{N(P)}$ (Theorem 2.15).

2.1. The category of diagrams. Categories of functors between a small category and a cofibrantly generated model category always admit cofibrantly generated model structures. We recall how to define the class of generating (trivial) cofibrations in the case of $\operatorname{Diag}_{P}$. We also exhibit an adjunction $\operatorname{Diag}_{P} \leftrightarrow \operatorname{Top}_{N(P)}$.

Definition 2.1. Let $\Delta^{\varphi} \in R(P)$ be a non-degenerate simplex and $K$ a simplicial set. Define $K^{\Delta^{\varphi}}: R(P)^{o p} \rightarrow$ sSet as the following functor

$$
\begin{aligned}
K^{\Delta^{\varphi}}: R(P)^{o p} & \rightarrow \text { sSet } \\
\Delta^{\psi} & \mapsto \begin{cases}K & \text { if } \Delta^{\psi} \subseteq \Delta^{\varphi} \\
\emptyset & \text { else }\end{cases}
\end{aligned}
$$

Where, for $\Delta^{\psi_{1}} \subseteq \Delta^{\psi_{2}} \subseteq \Delta^{\varphi}$, the maps $K^{\Delta^{\varphi}}\left(\Delta^{\psi_{1}} \rightarrow \Delta^{\psi_{2}}\right)$ are defined as $I d_{K}: K \rightarrow K$.

Proposition 2.2. The category $\operatorname{Diag}_{P}$ together with the following classes of maps is a cofibrantly generated model category.

- Fibrations are the maps $f: F \rightarrow G$ such that for all $\Delta^{\varphi} \in R(P), f\left(\Delta^{\varphi}\right): F\left(\Delta^{\varphi}\right) \rightarrow G\left(\Delta^{\varphi}\right)$ is a Kan fibration.

- Weak-equivalences are the maps $f: F \rightarrow G$ such that for all $\Delta^{\varphi} \in R(P), f\left(\Delta^{\varphi}\right): F\left(\Delta^{\varphi}\right) \rightarrow$ $G\left(\Delta^{\varphi}\right)$ is a weak-equivalence in the Kan model structure on sSet.

- Cofibrations are the maps with the left lifting property against trivial fibrations.

The sets of generating cofibrations and trivial cofibrations are the following

- $I=\left\{\left(\partial\left(\Delta^{n}\right) \rightarrow \Delta^{n}\right)^{\Delta^{\varphi}} \mid 0 \leq n, \Delta^{\varphi} \in R(P)\right\}$

- $J=\left\{\left(\Lambda_{k}^{n} \rightarrow \Delta^{n}\right)^{\Delta^{\varphi}} \mid 0 \leq k \leq n, n>0, \Delta^{\varphi} \in R(P)\right\}$ 
Proof. This is simply the projective model structure on $\operatorname{Fun}\left(R(P)^{o p}\right.$, sSet). See Hir03, Theorem 11.6.1].

Definition 2.3. We define the bi-functor $-\otimes-: \operatorname{sSet} \times \operatorname{sSet}_{P} \rightarrow \operatorname{sSet}_{P}$ as follows :

$$
\begin{aligned}
-\otimes-: \operatorname{sSet} \times \operatorname{sSet}_{P} & \rightarrow \operatorname{sSet}_{P} \\
K \otimes\left(X, \varphi_{X}\right) & \mapsto\left(K \times X, \varphi_{X} \circ p r_{X}\right) \\
(f: K \rightarrow L) \otimes\left(g:\left(X, \varphi_{X}\right) \rightarrow\left(Y, \varphi_{Y}\right)\right) & \mapsto f \times g: K \otimes\left(X, \varphi_{X}\right) \rightarrow L \otimes\left(Y, \varphi_{Y}\right)
\end{aligned}
$$

Remark 2.4. We will also write $-\otimes-$ for the bi-functor Top $\times \operatorname{Top}_{N(P)} \rightarrow \operatorname{Top}_{N(P)}$ defined in a similar way. The conflict of notation will not be problematic because, thanks to the classical result of J. Milnor [Mil57, one has $\left\|K \otimes\left(X, \varphi_{X}\right)\right\|_{N(P)} \simeq\|K\| \otimes\left\|\left(X, \varphi_{X}\right)\right\|_{N(P)}$, . if either $K$ or $X$ has only finitely many non-degenerate simplices.

Definition 2.5. Let $\mathcal{C} \subset R(P)^{o p} \times R(P)$ be the full subcategory whose objects are of the form $\left(\Delta^{\varphi}, \Delta^{\psi}\right)$ with $\Delta^{\psi} \subseteq \Delta^{\varphi}$. Define the functor $-\otimes R(P)$ as follows

$$
\begin{aligned}
-\otimes R(P): \operatorname{Diag}_{P} & \rightarrow \operatorname{Fun}\left(\mathcal{C}, \operatorname{sSet}_{P}\right) \\
F & \mapsto\left\{\begin{array}{ccc}
\mathcal{C} & \rightarrow & \operatorname{sSet}_{P} \\
\left(\Delta^{\varphi}, \Delta^{\psi}\right) & \mapsto & F\left(\Delta^{\varphi}\right) \otimes \Delta^{\psi}
\end{array}\right.
\end{aligned}
$$

Define the functor Colim: $\operatorname{Diag}_{P} \rightarrow \operatorname{Top}_{N(P)}$ as follows

$$
\begin{aligned}
& \text { Colim: } \operatorname{Diag}_{P} \rightarrow \operatorname{Top}_{N(P)} \\
& F \mapsto \operatorname{colim}_{\mathcal{C}}\|F \otimes R(P)\|_{N(P)}
\end{aligned}
$$

Proposition 2.6. The functor Colim: $\operatorname{Diag}_{P} \rightarrow \operatorname{Top}_{N(P)}$ is left adjoint to the functor $D: \operatorname{Top}_{N(P)} \rightarrow$ $\operatorname{Diag}_{P}$.

Proof. Let $F \in \operatorname{Diag}_{P}$ be a functor and $\left(X, \varphi_{X}\right)$ be a strongly stratified space over $P$. A map $f: F \rightarrow D\left(X, \varphi_{X}\right)$ is the coherent data of a map of simplicial sets

$$
f_{\Delta^{\varphi}}: F\left(\Delta^{\varphi}\right) \rightarrow \operatorname{Sing}\left(\mathcal{C}_{N(P)}^{0}\left(\left\|\Delta^{\varphi}\right\|_{N(P)},\left(X, \varphi_{X}\right)\right)\right),
$$

for all $\Delta^{\varphi} \in R(P)$. By the adjunction $(\|-\|$, Sing), this is equivalent to the coherent data of maps

$$
\left\|F\left(\Delta^{\varphi}\right)\right\| \rightarrow \mathcal{C}_{N(P)}^{0}\left(\left\|\Delta^{\varphi}\right\|_{N(P)},\left(X, \varphi_{X}\right)\right)
$$

By Lemma 2.7, and using the fact that $\left\|F\left(\Delta^{\varphi}\right)\right\| \otimes\left\|\Delta^{\varphi}\right\|_{N(P)} \simeq\left\|F\left(\Delta^{\varphi}\right) \otimes \Delta^{\varphi}\right\|_{N(P)}$ this is equivalent to the coherent data of maps

$$
\left\|F\left(\Delta^{\varphi}\right) \otimes \Delta^{\varphi}\right\|_{N(P)} \rightarrow\left(X, \varphi_{X}\right)
$$

Which corresponds to a map $\operatorname{Colim}(F) \rightarrow\left(X, \varphi_{X}\right)$.

Lemma 2.7. For all $\Delta^{\varphi} \in R(P)$, the functor $-\otimes\left\|\Delta^{\varphi}\right\|_{N(P)}$ is left adjoint to the functor $\mathcal{C}_{N(P)}^{0}\left(\left\|\Delta^{\varphi}\right\|_{N(P)},-\right)$.

Proof. This follows from the definition of $\mathcal{C}_{N(P)}^{0}$ and the fact that Top is cartesian closed (recall that Top stands for the category of $\Delta$-generated space, see Dug03). 
2.2. The model category $\operatorname{Top}_{N(P)}$. In this section, we prove the following theorem.

Theorem 2.8. There exists a cofibrantly generated model structure on Top $p_{N(P)}$ where a map $f:\left(X, \varphi_{X}\right) \rightarrow\left(Y, \varphi_{Y}\right)$ is

- a fibration if $D(f)$ is a fibration,

- a weak-equivalence if $D(f)$ is a weak-equivalence,

- a cofibration if it has the left lifting property against all trivial fibrations.

The set of generating cofibrations and trivial cofibrations are

- $I=\left\{\left\|\partial\left(\Delta^{n}\right) \otimes \Delta^{\varphi} \rightarrow \Delta^{n} \otimes \Delta^{\varphi}\right\|_{N(P)} \mid n \geq 0, \Delta^{\varphi} \in R(P)\right\}$

- $J=\left\{\left\|\Lambda_{k}^{n} \otimes \Delta^{\varphi} \rightarrow \Delta^{n} \otimes \Delta^{\varphi}\right\|_{N(P)} \mid 0 \leq k \leq n n>0, \Delta^{\varphi} \in R(P)\right\}$

Remark 2.9. A weak-equivalence in $\operatorname{Top}_{N(P)}$ is a map, $f:\left(X, \varphi_{X}\right) \rightarrow\left(Y, \varphi_{Y}\right)$ inducing weakequivalences $D(f)\left(\Delta^{\varphi}\right): D\left(X, \varphi_{X}\right)\left(\Delta^{\varphi}\right) \rightarrow D\left(Y, \varphi_{Y}\right)\left(\Delta^{\varphi}\right)$ for all $\Delta^{\varphi} \in N(P)$. Equivalently, $f$ is a weak-equivalence, if and only if it induces isomorphisms of functors $s \pi_{n}(f): s \pi_{n}\left(\left(X, \varphi_{X}\right), \phi\right) \rightarrow$ $s \pi_{n}\left(\left(Y, \varphi_{Y}\right), f \circ \phi\right)$, for all pointings of $X$ and for all $n$.

Proof. By lemma 2.10, [HKRS17, Corollary 3.3.4] applies and we only need to show that any filtered map having the left lifting property against any fibration is a weak-equivalence (in the sense of Theorem 2.8). Let $f:\left(X, \varphi_{X}\right) \rightarrow\left(Y, \varphi_{Y}\right)$ be such a filtered map. By lemma 2.11, $f$ admits a factorization of the form

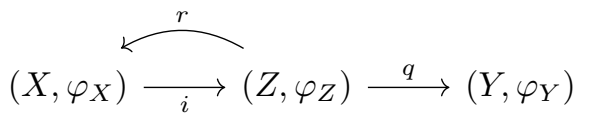

Consider the following lifting problem

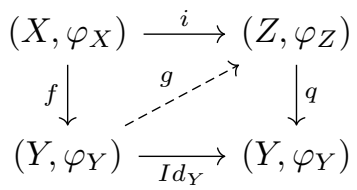

By lemma 2.11, $q$ is a fibration, hence there exists a lift $g:\left(Y, \varphi_{Y}\right) \rightarrow\left(Z, \varphi_{Z}\right)$. Fixing some pointing of $X, \phi$ and passing to stratified homotopy groups, we get

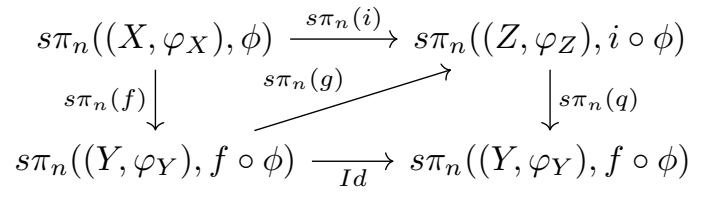

Since $I d$ and $s \pi_{n}(i)$ are isomorphisms (By lemma 2.11), so is $s \pi_{n}(g)$ since it admits left and right inverses. But then, $s \pi_{n}(f)$ is an isomorphism with inverse $\left(s \pi_{n}(i)\right)^{-1} s \pi_{n}(g)$. By Remarque 2.9, this implies that $f$ is a weak-equivalence, which concludes the proof of the first part of theorem 2.8. The set of generating cofibrations and trivial cofibrations are then obtained by applying the functor Colim to the corresponding generating set in $\operatorname{Diag}_{P}$.

Lemma 2.10. The model category $\operatorname{Diag}_{P}$ is accessible, and the category $T o p_{N(P)}$ is locally presentable.

Proof. The model category $\operatorname{Diag}_{P}$ is cofibrantly generated, and locally presentable so it is accessible (see [HKRS17]). In this text, Top is the category of $\Delta$-generated spaces, which is locally presentable (see $\left[\right.$ Dug03). Since $\operatorname{Top}_{N(P)}=\operatorname{Top} /\|N(P)\|$, it is also locally presentable. 
Lemma 2.11. Let $f:\left(X, \varphi_{X}\right) \rightarrow\left(Y, \varphi_{Y}\right)$ be a filtered map. There exists a strongly filtered space, $\left(Z, \varphi_{Z}\right)$, a fibration $q:\left(Z, \varphi_{Z}\right) \rightarrow\left(Y, \varphi_{Y}\right)$, a filtered map $i:\left(X, \varphi_{X}\right) \rightarrow\left(Z, \varphi_{Z}\right)$ and a filtered map $r:\left(Z, \varphi_{Z}\right) \rightarrow\left(X, \varphi_{X}\right)$ such that

- $f=q \circ i$,

- $r \circ i=I d_{X}$ and $i \circ r$ is filtered homotopic to $I d_{Z}$.

Proof. Let $f:\left(X, \varphi_{X}\right) \rightarrow\left(Y, \varphi_{Y}\right)$ be a filtered map. Define the topological space $Z$ as the following subspace of $X \times Y^{[0,1]}$ :

$$
Z=\left\{(x, \gamma) \in X \times Y^{[0,1]} \mid f(x)=\gamma(0), \varphi_{Y}(\gamma(t))=\varphi_{X}(x), \forall t \in[0,1]\right\},
$$

and $\varphi_{Z}: Z \rightarrow\|N(P)\|$ as the following composition

$$
Z \longleftrightarrow X \times Y^{[0,1]} \stackrel{p r_{X}}{\longrightarrow} X \stackrel{\varphi_{X}}{\longrightarrow}\|N(P)\|
$$

Define $i, q$ and $r$ as follows

$$
\begin{aligned}
i:\left(X, \varphi_{X}\right) & \rightarrow\left(Z, \varphi_{Z}\right) \\
x & \mapsto(x, t \mapsto f(x)) \\
q:\left(Z, \varphi_{Z}\right) & \rightarrow\left(Y, \varphi_{Y}\right) \\
(x, \gamma) & \mapsto \gamma(1) \\
r:\left(Z, \varphi_{Z}\right) & \rightarrow\left(X, \varphi_{X}\right) \\
(x, \gamma) & \mapsto x
\end{aligned}
$$

One has $f=q \circ i$ and $r \circ i=I d_{X}$, and the filtered map

$$
\begin{aligned}
\left(Z \times[0,1], \varphi_{Z} \circ p r_{Z}\right) & \rightarrow\left(Z, \varphi_{Z}\right) \\
((x, \gamma), s) & \mapsto(x,(t \mapsto \gamma(s t))
\end{aligned}
$$

is a filtered homotopy between $i \circ r$ and $I d_{Z}$. The only thing left to check is that $q$ is a fibration. Consider the following lifting problem

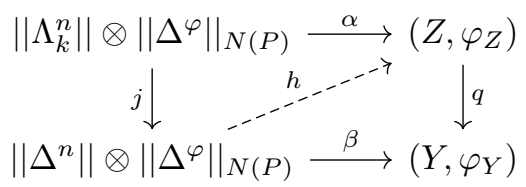

The inclusion $\left\|\Lambda_{k}^{n}\right\| \rightarrow\left\|\Delta^{n}\right\|$ admits a deformation retract $\left\|\Delta^{n}\right\| \rightarrow\left\|\Lambda_{k}^{n}\right\|$. Taking the product with $I d_{\Delta^{\varphi}}$, one gets a filtered retract of $j, s:\left\|\Delta^{n}\right\| \otimes\left\|\Delta^{\varphi}\right\|_{N(P)} \rightarrow\left\|\Lambda_{k}^{n}\right\| \otimes\left\|\Delta^{\varphi}\right\|_{N(P)}$ together with a filtered homotopy between $j \circ s$ and $I d_{\left\|\Delta^{n}\right\| \otimes\left\|\Delta^{\varphi}\right\|_{N(P)}}$.

$$
H:[0,1] \otimes\left\|\Delta^{n}\right\| \otimes\left\|\Delta^{\varphi}\right\|_{N(P)} \rightarrow\left\|\Delta^{n}\right\| \otimes\left\|\Delta^{\varphi}\right\|_{N(P)} .
$$

Fix some continuous map $d:\left\|\Delta^{n}\right\| \rightarrow \mathbb{R}_{\geq 0}$ such that $h^{-1}(0)=\left\|\Lambda_{k}^{n}\right\|$ (for example, the distance to $\left.\left\|\Lambda_{k}^{n}\right\|\right)$, and for $a \in\left\|\Lambda_{k}^{n}\right\| \otimes\left\|\Delta^{\varphi}\right\|_{N(P)}$, write $\alpha(a)=\left(\alpha_{X}(a), \alpha_{Y}(a)\right) \in Z \subset X \times Y^{[0,1]}$. With those 
notations, we will define $h$ as $\left(h_{X}, h_{Y}\right)$. Define $h_{X}$ as $\alpha_{X} \circ s$, and $h_{Y}$ as follows

$$
\begin{aligned}
& h_{Y}:\left\|\Delta^{n}\right\| \otimes\left\|\Delta^{\varphi}\right\|_{N(P)} \rightarrow Y^{[0,1]} \\
& a \mapsto\left\{\begin{array}{cl}
{[0,1]} & \rightarrow \\
t & \mapsto \begin{cases}\alpha_{Y}(s(a))(t(1+d(a))) & \text { if } 0 \leq t \leq \frac{1}{1+d(a)} \\
\beta\left(H\left(a, \frac{1+d(a)}{d(a)}\left(t-\frac{1}{1+d(a)}\right)\right)\right. & \text { if } \frac{1}{1+d(a)}<t \leq 1\end{cases}
\end{array}\right.
\end{aligned}
$$

One checks that $h=\left(h_{X}, h_{Y}\right)$ is indeed a lift for the problem (2).

2.3. Quillen equivalence between $\operatorname{Top}_{N(P)}$ and $\operatorname{Diag}_{P}$. In this section, we prove the following theorem.

Theorem 2.12. The adjunction

$$
\text { Colim: } \operatorname{Diag}_{P} \leftrightarrow \operatorname{Top}_{N(P)}: D
$$

is a Quillen equivalence.

Proof. By definition of the model structure on $\operatorname{Top}_{N(P)}, D$ preserves fibrations and weak-equivalences, hence (Colim, $D$ ) is a Quillen adjunction. To show that it is a Quillen equivalence, one needs to prove that for any cofibrant object $F$ in $\operatorname{Diag}_{P}$, the unit $\eta_{F}: F \rightarrow D(\operatorname{Colim}(F))$ is a weak-equivalence. By definition of the model structure on $\operatorname{Diag}_{P}$, this amount to showing that for all $\Delta^{\varphi} \in R(P)$, the map

$$
F\left(\Delta^{\varphi}\right) \rightarrow \operatorname{Sing}\left(\mathcal{C}_{N(P)}^{0}\left(\left\|\Delta^{\varphi}\right\|_{N(P)}, \operatorname{Colim}(F)\right)\right)
$$

is a weak-equivalence of simplicial sets. By adjunction, this is equivalent to showing that the map

$$
\left\|F\left(\Delta^{\varphi}\right)\right\| \rightarrow \mathcal{C}_{N(P)}^{0}\left(\left\|\Delta^{\varphi}\right\|_{N(P)}, \operatorname{Colim}(F)\right)
$$

is a weak-equivalence of topological spaces. We will factor this map as follows

$$
\left\|F\left(\Delta^{\varphi}\right)\right\| \stackrel{f}{\longrightarrow} \mathcal{C}_{N(P)}^{0}\left(\left\|\Delta^{\varphi}\right\|_{N(P)},\left\|F\left(\Delta^{\varphi}\right)\right\| \otimes\left\|\Delta^{\varphi}\right\|_{N(P)}\right) \stackrel{g}{\longrightarrow} \mathcal{C}_{N(P)}^{0}\left(\left\|\Delta^{\varphi}\right\|_{N(P)}, \operatorname{Colim}(F)\right)
$$

Where $f$ is the obvious map sending $x \in\left\|F\left(\Delta^{\varphi}\right)\right\|$ to $t \mapsto(x, t)$, and $g$ comes from the map

$$
i_{\Delta^{\varphi}}:\left\|F\left(\Delta^{\varphi}\right)\right\| \otimes\left\|\Delta^{\varphi}\right\|_{N(P)} \rightarrow \operatorname{colim}\left\|F\left(\Delta^{\varphi}\right)\right\| \otimes\left\|\Delta^{\psi}\right\|_{N(P)}=\operatorname{Colim}(F)
$$

The map $f$ admits a retraction, $r$, given by picking a point $t_{0} \in\left\|\Delta^{\varphi}\right\|_{N(P)}$, evaluating at $t_{0}$ then projecting the result onto $\left\|F\left(\Delta^{\varphi}\right)\right\|$. Choosing some deformation retract from $\left\|\Delta^{n}\right\|$ to $\left\{t_{0}\right\}$, where $\Delta^{n}$ is the non-stratified simplex underlying $\Delta^{\varphi}$, then gives a homotopy between $f \circ r$ and $I d$. In particular, $f$ is a homotopy equivalence. We will show that $g$ is a homeomorphism, which will conclude the proof. First, notice that

$$
\operatorname{Colim}(F)=\bigcup_{\Delta^{\varphi} \in R(P)} \operatorname{Im}\left(i_{\Delta^{\varphi}}\right)=\bigcup_{\Delta^{\varphi} \in R(P)} i_{\Delta^{\varphi}}\left(\left\|F\left(\Delta^{\varphi}\right)\right\| \otimes \operatorname{Int}\left(\left\|\Delta^{\varphi}\right\|_{N(P)}\right)\right),
$$

where Int $\left(\left\|\Delta^{\varphi}\right\|_{N(P)}\right)$ means the set of points of $\left\|\Delta^{\varphi}\right\|_{N(P)}$ which are not in the boundary $\left\|\partial\left(\Delta^{\varphi}\right)\right\|_{N(P)}$. In particular, this implies that for all $\Delta^{\varphi} \in R(P)$, the following diagram is cartesian

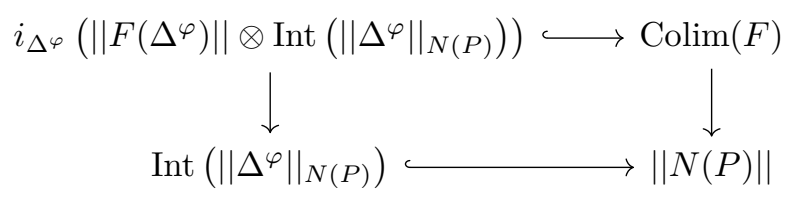


Let $\phi:\left\|\Delta^{\varphi}\right\|_{N(P)} \rightarrow \operatorname{Colim}(F)$ be a filtered map. Write $\phi_{\text {Int }}$ its restriction to Int $\left(\left\|\Delta^{\varphi}\right\|_{N(P)}\right)$. Since the square (3) is cartesian, the map $\phi_{\text {Int }}$ factors through $i_{\Delta^{\varphi}}\left(\| F\left(\Delta^{\varphi} \| \otimes \operatorname{Int}\left(\left\|\Delta^{\varphi}\right\|_{N(P)}\right)\right)\right.$. In particular, $\phi$ must take value in the closure

$$
\overline{i_{\Delta^{\varphi}}\left(\left\|F\left(\Delta^{\varphi}\right)\right\| \otimes \operatorname{Int}\left(\left\|\Delta^{\varphi}\right\|_{N(P)}\right)\right)} \subset \operatorname{Colim}(F)
$$

By lemma 2.13 , this closure is precisely the image of $\left\|F\left(\Delta^{\varphi}\right)\right\| \otimes\left\|\Delta^{\varphi}\right\|_{N(P)}$ in $\operatorname{Colim}(F)$, which is homeomorphic to $\left\|F\left(\Delta^{\varphi}\right)\right\| \otimes\left\|\Delta^{\varphi}\right\|_{N(P)}$. In conclusion, all filtered maps $\phi: \Delta^{\varphi} \rightarrow \operatorname{Colim}(F)$ factor through the subspace $\left\|F\left(\Delta^{\varphi}\right)\right\| \otimes\left\|\Delta^{\varphi}\right\|_{N(P)} \subset \operatorname{Colim}(F)$, and so the inclusion $g$ is in fact a homeomorphism.

Lemma 2.13. Let $F$ be a cofibrant object of $\operatorname{Diag}_{P}$. Then, for all $\Delta^{\varphi} \in R(P)$, the map

$$
i_{\Delta \varphi}:\left\|F\left(\Delta^{\varphi}\right)\right\| \otimes\left\|\Delta^{\varphi}\right\|_{N(P)} \rightarrow \operatorname{Colim}(F)
$$

is a homeomorphism onto its image, and its image is closed in Colim $(F)$.

Proof. Given a cofibrant diagram $F$, the space underlying $\operatorname{Colim}(F)$ is the realisation of the simplicial set colim $F \otimes R(P)$. In particular, it is enough to show that the map of simplicial sets $F\left(\Delta^{\varphi}\right) \otimes \Delta^{\varphi^{\mathcal{C}}} \rightarrow \operatorname{colim}_{\mathcal{C}} F \otimes R(P)$ is a monomorphism. First notice that if a diagram is cofibrant, then for any inclusion $\Delta^{\varphi} \subset \Delta^{\psi}$, the map $F\left(\Delta^{\psi}\right) \rightarrow F\left(\Delta^{\varphi}\right)$ is a monomorphism. Indeed, given any trivial Kan fibration $q: K \rightarrow L$, one proves that $F\left(\Delta^{\psi}\right) \rightarrow F\left(\Delta^{\varphi}\right)$ admits the left lifting property against $q$ by considering the lifting property of $\emptyset \rightarrow F$ with respect to the trivial fibration $G \rightarrow H$ where $G$ and $H$ are the diagrams respectively defined as :

$$
G\left(\Delta^{\mu}\right)=\left\{\begin{array}{ll}
L & \text { if } \Delta^{\mu} \subsetneq \Delta^{\varphi} \\
K & \text { else }
\end{array} \text { and } H\left(\Delta^{\mu}\right)= \begin{cases}L & \text { if } \Delta^{\mu} \subseteq \Delta^{\varphi} \\
K & \text { else }\end{cases}\right.
$$

We can then conclude by using Remark A.4 and Lemma A.5 to apply Proposition A.2.

2.4. Quillen equivalence between the categories of filtered and strongly filtered spaces. The goal of this section is to prove Theorem 2.15] which, together with Theorem 2.12 provides a proof of Theorem 3 ,

Definition 2.14. Let $D_{P}$ : $\operatorname{Top}_{P} \rightarrow \operatorname{Diag}_{P}$ be the composition of $D$ with $-\times_{P}\|N(P)\|$, where $-\times_{P}\|N(P)\|$ is the functor from Definition 1.23 .

Theorem 2.15. There exists a cofibrantly generated model structure on Top $p_{P}$ where a map $f:\left(X, \varphi_{X}\right) \rightarrow$ $\left(Y, \varphi_{Y}\right)$ is

- a fibration if $D_{P}(f)$ is a fibration,

- a weak-equivalence if $D_{P}(f)$ is a weak-equivalence,

- a cofibration if it has the left lifting property against all trivial fibrations.

The set of generating cofibrations and trivial cofibrations are

- $I=\left\{\left\|\partial\left(\Delta^{n}\right) \otimes \Delta^{\varphi} \rightarrow \Delta^{n} \otimes \Delta^{\varphi}\right\|_{P} \mid n \geq 0, \Delta^{\varphi} \in R(P)\right\}$

- $J=\left\{\left\|\Lambda_{k}^{n} \otimes \Delta^{\varphi} \rightarrow \Delta^{n} \otimes \Delta^{\varphi}\right\|_{P} \mid 0 \leq k \leq n n>0, \Delta^{\varphi} \in R(P)\right\}$

Furthermore, the adjunction

$$
\varphi_{P} \circ-: \operatorname{Top}_{N(P)} \leftrightarrow \operatorname{Top}_{P}:-\times_{P}\|N(P)\|
$$

is a Quillen-equivalence. 
Proof. The proof of Theorem 2.8 generalizes directly to show the existence of the model structure. Let $f:\left(X, \varphi_{X}\right) \rightarrow\left(Y, \varphi_{Y}\right)$ be a morphism of $\operatorname{Top}_{P}$. It is a (trivial) fibration if and only if $D_{P}(f): D_{P}\left(X, \varphi_{X}\right) \rightarrow D_{P}\left(Y, \varphi_{Y}\right)$ is a (trivial) fibration. The latter is true if and only if $f \times_{P}\|N(P)\|: X \times_{P}\|N(P)\| \rightarrow Y \times_{P}\|N(P)\|$ is a (trivial) fibration of $\operatorname{Top}_{N(P)}$. In particular, $\left(\varphi_{P} \circ-,-\times_{P}\|N(P)\|\right)$ is a Quillen-adjunction. Let $\left(X, \varphi_{X}\right) \in \operatorname{Top}_{N(P)}$ be a strongly filtered space, $\left(Y, \varphi_{Y}\right) \in \operatorname{Top}_{P}$ be a filtered space, and $f:\left(X, \varphi_{P} \circ \varphi_{X}\right) \rightarrow\left(Y, \varphi_{Y}\right)$ be a filtered map. We need to show that $f$ is a weak-equivalence of $\operatorname{Top}_{P}$ if and only if its adjoint map is a weak-equivalence:

$$
\tilde{f}:\left(X, \varphi_{X}\right) \rightarrow\left(Y, \varphi_{Y}\right) \times_{P}\|N(P)\| .
$$

Notice that $\tilde{f}$ factors as

$$
\left(X, \varphi_{X}\right) \stackrel{\epsilon_{X}}{\longrightarrow}\left(X, \varphi_{P} \circ \varphi_{X}\right) \times_{P}\|N(P)\| \stackrel{f \times_{P}\|N(P)\|}{\longrightarrow}\left(Y, \varphi_{Y}\right) \times_{P}\|N(P)\|
$$

By definition $f \times{ }_{P}\|N(P)\|$ is a weak-equivalence of $\operatorname{Top}_{N(P)}$ if and only if $D\left(f \times{ }_{P}\|N(P)\|\right)$ is a weak-equivalence of $\operatorname{Diag}_{P}$ if and only if $f$ is a weak equivalence of $\operatorname{Top}_{P}$. In particular, it is enough to show that $\epsilon_{X}$ is a weak-equivalence of $\operatorname{Top}_{N(P)}$. Consider the following commutative diagram

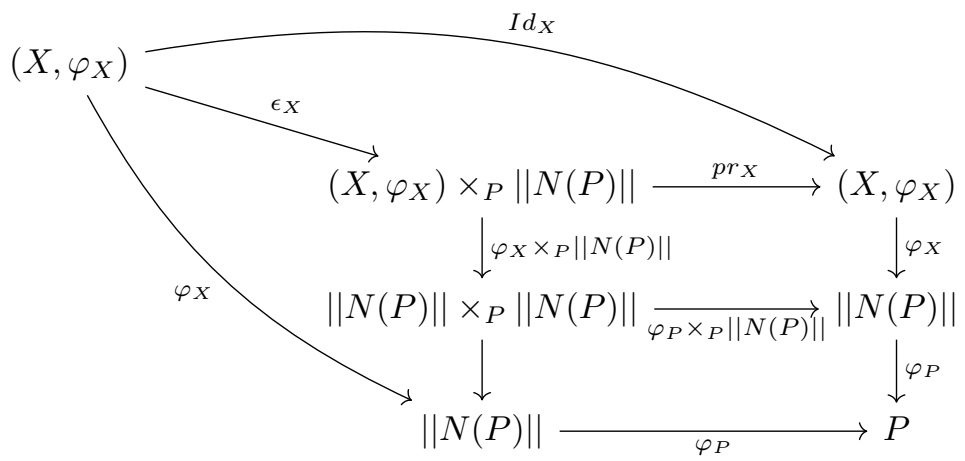

All three squares are pullback square. By Lemma 2.16 $\varphi_{P}$ is a trivial fibration in $\operatorname{Top}_{P}$. Since $-\times_{P}\|N(P)\|$ preserves trivial fibrations, $\varphi_{P} \times_{P}\|N(P)\|$ is a trivial fibration in $\operatorname{Top}_{N(P)}$, and since the top square is a pullback square, so is $p r_{X}$. But then, by two out of three, $\epsilon_{X}$ is a weak-equivalence in $\operatorname{Top}_{N(P)}$.

Lemma 2.16. The filtered map

$$
\varphi_{P}:\left(\|N(P)\|, \varphi_{P}\right) \rightarrow\left(P, I d_{P}\right)
$$

is a trivial fibration in $\operatorname{Top}_{P}$.

Proof. We need to prove that for all $\Delta^{\varphi} \in R(P)$, the map

$$
\operatorname{Sing}\left(\mathcal{C}_{P}^{0}\left(\left\|\Delta^{\varphi}\right\|_{P},\left(\|N(P)\|, \varphi_{P}\right)\right)\right) \rightarrow \operatorname{Sing}\left(\mathcal{C}_{P}^{0}\left(\left\|\Delta^{\varphi}\right\|_{P},\left(P, I d_{P}\right)\right)\right)
$$

Is a trivial fibration in sSet. Using the Quillen-equivalence given by $(\|-\|$, Sing), this amounts to showing that the map

$$
\mathcal{C}_{P}^{0}\left(\left\|\Delta^{\varphi}\right\|_{P},\left(\|N(P)\|, \varphi_{P}\right)\right) \rightarrow \mathcal{C}_{P}^{0}\left(\left\|\Delta^{\varphi}\right\|_{P},\left(P, I d_{P}\right)\right)
$$

is a trivial fibration in Top. First notice that $\mathcal{C}_{P}^{0}\left(\left\|\Delta^{\varphi}\right\|_{P},\left(P, I d_{P}\right)\right) \simeq\{*\}$, that is, there is only a single filtered map $\left\|\Delta^{\varphi}\right\|_{P} \rightarrow\left(P, I d_{P}\right)$, given by the composition

$$
\left\|\Delta^{\varphi}\right\|_{P} \longleftrightarrow\left(\|N(P)\|, \varphi_{P}\right) \stackrel{\varphi_{P}}{\longrightarrow}\left(P, I d_{P}\right)
$$


In particular, since every topological space is fibrant, the map (4) is a fibration. To show that it is a weak-equivalence, it remains to show that $\mathcal{C}_{P}^{0}\left(\left\|\Delta^{\varphi}\right\|_{P},\left(\|N(P)\|, \varphi_{P}\right)\right)$ is contractible. Write $\Delta^{\varphi}=$ $\left\{p_{0}<\cdots<p_{n}\right\} \subset N(P)$. First notice that any filtered map $f:\left\|\Delta^{\varphi}\right\|_{P} \rightarrow\left(\|N(P)\|, \varphi_{P}\right)$ must factor through $\varphi_{P}^{-1}\left(\left\{p_{0}, \ldots, p_{n}\right\}\right)$. Indeed, since $f$ is filtered, one must have for any $x \in\left\|\Delta^{\varphi}\right\|_{P}$, $\varphi_{P}(f(x))=\varphi_{P}(x) \in\left\{p_{0}, \ldots, p_{n}\right\} \subset P$. And so, by Lemma 2.17, we get a homotopy equivalence

$$
\mathcal{C}_{P}^{0}\left(\left\|\Delta^{\varphi}\right\|_{P},\left(\|N(P)\|, \varphi_{P}\right)\right) \sim \mathcal{C}_{P}^{0}\left(\left\|\Delta^{\varphi}\right\|_{P},\left\|\Delta^{\varphi}\right\|_{P}\right) .
$$

But the latter is contractible by lemma 2.18, which concludes the proof.

Lemma 2.17. Let $\Delta^{\varphi}=\left\{p_{0}<\cdots<p_{n}\right\}$ be a (non-degenerate) simplex of $N(P)$. The inclusion

$$
\left\|\Delta^{\varphi}\right\|_{P} \hookrightarrow \varphi_{P}^{-1}\left(\left\{p_{0}, \ldots, p_{n}\right\}\right)
$$

is a filtered homotopy equivalence.

Proof. Let $x=\left(\Delta^{\psi},\left(t_{0}, \ldots, t_{m}\right)\right)$ be a point in $\varphi_{P}^{-1}\left(\left\{p_{0}, \ldots, p_{n}\right\}\right) \subset\|N(P)\|$, with $\Delta^{\psi}=\left\{q_{0}<\right.$ $\left.\cdots<q_{m}\right\}$. Define $j=\max \left\{i \mid t_{i} \neq 0\right\}$, then $\varphi_{P}(x)=q_{j}$, and so $q_{j} \in\left\{p_{0}, \ldots, p_{n}\right\}$. For any non-degenerate simplex $\Delta^{\psi}=\left\{q_{0}<\cdots<q_{m}\right\}$, write $I^{\psi} \subset\{0, \ldots, m\}$ for the set of $i$ satisfying $q_{i} \in\left\{p_{0}, \ldots, p_{n}\right\}$. Define the following homotopy

$$
\begin{aligned}
H^{\psi}:\left\|\Delta^{\psi}\right\|_{P} \cap \varphi_{P}^{-1}\left(\left\{p_{0}<\cdots<p_{n}\right\}\right) \times[0,1] & \rightarrow\left\|\Delta^{\psi}\right\|_{P} \cap \varphi_{P}^{-1}\left(\left\{p_{0}<\cdots<p_{n}\right\}\right) \\
\left(\left(t_{0}, \ldots, t_{m}\right), s\right) & \mapsto\left(H_{1}^{\psi}\left(\left(t_{0}, \ldots, t_{m}\right), s\right), \ldots, H_{m}^{\psi}\left(\left(t_{0}, \ldots, t_{m}\right), s\right)\right)
\end{aligned}
$$

Where, for $0 \leq i \leq m, H_{i}^{\psi}$ is defined as follows

$$
\begin{aligned}
H_{i}^{\psi}:\left\|\Delta^{\psi}\right\|_{P} \cap \varphi_{P}^{-1}\left(\left\{p_{0}, \ldots, p_{n}\right\}\right) \times[0,1] & \rightarrow[0,1] \\
\left(\left(t_{0}, \ldots, t_{m}\right), s\right) & \mapsto\left\{\begin{array}{cl}
t_{i}(1-s) & \text { if } i \notin I^{\psi} \\
t_{i}\left(1+s\left(\frac{\sum_{j \notin I} t_{j}}{\sum_{j \in I} t_{j}}\right)\right) & \text { if } i \in I^{\psi}
\end{array}\right.
\end{aligned}
$$

One checks that $\sum_{i} H_{i}^{\psi}=1$, and that the $H_{i}^{\psi}$ are all non-negative, so that $H^{\psi}$ is well-defined. Furthermore, by construction, $H^{\psi}$ is a filtered map. In particular, its image lies in $\varphi_{P}^{-1}\left(\left\{p_{0}, \ldots, p_{n}\right\}\right)$. Finally, $H^{\psi}$ is a homotopy between the identity, and a composition

$$
\left\|\Delta^{\psi}\right\|_{P} \cap \varphi_{P}^{-1}\left(\left\{p_{0}, \ldots, p_{n}\right\}\right) \rightarrow\left\|\Delta^{\psi}\right\|_{P} \cap\left\|\Delta^{\varphi}\right\|_{P} \hookrightarrow\left\|\Delta^{\psi}\right\|_{P} \cap \varphi_{P}^{-1}\left(\left\{p_{0}, \ldots, p_{n}\right\}\right)
$$

Let us show that we can glue the $H^{\psi}$ together. Let $\Delta^{\mu} \subset \Delta^{\psi}$ be two non-degenerate simplices of $N(P)$, and let $x \in\left\|\Delta^{\mu}\right\|_{P} \cap \varphi_{P}^{-1}\left(\left\{p_{0}, \ldots, p_{n}\right\}\right)$. Then, $x=\left(\Delta^{\psi},\left(t_{0}, \ldots, t_{m}\right)\right)=\left(\Delta^{\mu},\left(u_{0}, \ldots, u_{l}\right)\right)$, with $t_{\alpha(i)}=u_{i}$ for all $0 \leq i \leq l$, and $t_{i}=0$ if $i \notin \alpha\{0, \ldots, l\}$, where $\alpha: \Delta^{l} \rightarrow \Delta^{m}$ is the face map corresponding to the inclusion $\Delta^{\mu} \subset \Delta^{\psi}$. Furthermore, $I^{\psi} \cap \alpha(\{0, \ldots, l\})=\alpha\left(I^{\mu}\right)$. Let $s \in[0,1]$. For $i \in\{0, \ldots, l\}$, one computes :

- if $i \in I^{\mu}, H_{i}^{\mu}\left(\left(u_{0}, \ldots, u_{l}\right), s\right)=u_{i}\left(1+s\left(\frac{\sum_{j \notin I^{\mu}} u_{j}}{\sum_{j \in I^{\mu}} u_{j}}\right)\right)$. On the other hand, $\alpha(i) \in I^{\psi}$, and one has $H_{\alpha(i)}^{\psi}\left(\left(t_{0}, \ldots, t_{m}\right), s\right)=t_{\alpha(i)}\left(1+s\left(\frac{\sum_{j \notin I} t_{j}}{\sum_{j \in I} t_{j}}\right)\right)$. But since $t_{\alpha(j)}=u_{j}$ for all 
$j \in\{0, \ldots, l\}$, and $k \notin \alpha(\{0, \ldots, l\}) \Rightarrow t_{k}=0$, one can rewrite the latter equality as

$$
\begin{aligned}
H_{\alpha(i)}^{\psi}\left(\left(t_{0}, \ldots, t_{m}\right), s\right) & =t_{\alpha(i)}\left(1+s\left(\frac{\sum_{j \notin I^{\mu}} t_{\alpha(j)}}{\sum_{j \in I^{\mu}} t_{\alpha(j)}}\right)\right) \\
& =u_{i}\left(1+s\left(\frac{\sum_{j \notin I^{\mu}} u_{j}}{\sum_{j \in I^{\mu}} u_{j}}\right)\right) \\
& =H_{i}^{\mu}\left(\left(u_{0}, \ldots, u_{l}\right), s\right) .
\end{aligned}
$$

- if $i \notin I^{\mu}$, then $H_{i}^{\mu}\left(\left(u_{0}, \ldots, u_{l}\right), s\right)=u_{i}(1-s)$. On the other hand, $\alpha(i) \notin I^{\psi}$, and $H_{\alpha(i)}^{\psi}\left(\left(t_{0}, \ldots, t_{m}\right), s\right)=t_{\alpha(i)}(1-s)=u_{i}(1-s)=H_{i}^{\mu}\left(\left(u_{0}, \ldots, u_{l}\right), s\right)$.

In all cases, one has $H_{i}^{\mu}(x, s)=H_{\alpha(i)}^{\psi}(x, s)$. Furthermore, if $j \notin \alpha(\{0, \ldots, l\}), t_{j}=0$, and $H_{j}^{\psi}(x, s)=0$. In conclusion, we have $H^{\mu}(x, s)=H^{\psi}(x, s)$, and the $H^{\psi}$ are compatible with faces. Since $\|N(P)\|$ is a simplicial complex, this is enough to glue the $H^{\psi}$ together to obtain a map

$$
H: \varphi_{P}^{-1}\left(\left\{p_{0}, \ldots, p_{n}\right\}\right) \times[0,1] \rightarrow \varphi_{P}^{-1}\left(\left\{p_{0}, \ldots, p_{n}\right\}\right)
$$

In particular, $H$ provides a filtered homotopy between the identity and the composition

$$
\varphi_{P}^{-1}\left(\left\{p_{0}, \ldots, p_{n}\right\}\right) \rightarrow\left\|\Delta^{\varphi}\right\|_{P} \hookrightarrow \varphi_{P}^{-1}\left(\left\{p_{0}, \ldots, p_{n}\right\}\right)
$$

Lemma 2.18. Let $\Delta^{\varphi}$ be a (non-degenerate) simplex of $N(P)$. The space of filtered maps

$$
\mathcal{C}_{P}^{0}\left(\left\|\Delta^{\varphi}\right\|_{P},\left\|\Delta^{\varphi}\right\|_{P}\right)
$$

is contractible.

Proof. Define the contracting homotopy as follows

$$
\begin{aligned}
& \mathcal{C}_{P}^{0}\left(\left\|\Delta^{\varphi}\right\|_{P},\left\|\Delta^{\varphi}\right\|_{P}\right) \times[0,1] \rightarrow \mathcal{C}_{P}^{0}\left(\left\|\Delta^{\varphi}\right\|_{P},\left\|\Delta^{\varphi}\right\|_{P}\right) \\
& \left(\left(f:\left\|\Delta^{\varphi}\right\|_{P} \rightarrow\left\|\Delta^{\varphi}\right\|_{P}\right), s\right) \mapsto\left\{\begin{aligned}
\left\|\Delta^{\varphi}\right\|_{P} & \rightarrow\left\|\Delta^{\varphi}\right\|_{P} \\
\left(t_{0}, \ldots, t_{n}\right) & \mapsto s f\left(t_{0}, \ldots, t_{n}\right)+(1-s)\left(t_{0}, \ldots, t_{n}\right)
\end{aligned}\right.
\end{aligned}
$$

\section{A MOdel CATEgory For STRATified SPACES}

In section 2, we constructed a model structure on the category of filtered spaces over any poset. In this section, using a theorem of P. Cagne and P.-A. Melliès [CM17, Theorem 4.2], we "glue" all those model structures together in order to obtain a model structure on Strat (Theorem 3.6).

3.1. Comparing model structures between posets. For this section, fix some map of posets $\alpha: P \rightarrow Q$. 
Definition 3.1. Define the functor $\alpha_{*}$ : $\operatorname{Top}_{P} \rightarrow \operatorname{Top}_{Q}$, and $\alpha^{*}: \operatorname{Top}_{Q} \rightarrow \operatorname{Top}_{P}$ as follows

$$
\begin{aligned}
\alpha_{*}: \operatorname{Top}_{P} & \rightarrow \operatorname{Top}_{Q} \\
\left(X, P, \varphi_{X}\right) & \mapsto\left(X, Q, \alpha \circ \varphi_{X}\right) \\
\alpha^{*}: \operatorname{Top}_{Q} & \rightarrow \operatorname{Top}_{P} \\
\left(Y, Q, \varphi_{Y}\right) & \mapsto\left(Y \times_{Q} P, P, \alpha^{*} \varphi_{Y}\right)
\end{aligned}
$$

where $\alpha^{*} \varphi_{Y}$ is defined by the following pullback square

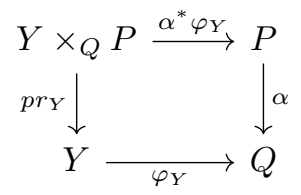

Proposition 3.2. The pair of functors

$$
\alpha_{*}: \operatorname{Top}_{P} \leftrightarrow \operatorname{Top}_{Q}: \alpha^{*}
$$

is a Quillen-adjunction. Furthermore, it is a Quillen-equivalence if and only if $\alpha$ is an isomorphism of posets.

Proof. It is an adjunction by construction. To show that it is a Quillen-adjunction, it is enough to show that the generating (trivial) cofibrations of $\operatorname{Top}_{P}$ are sent to (trivial) cofibrations. But

$$
\alpha_{*}\left(\left\|\partial\left(\Delta^{n}\right) \otimes \Delta^{\varphi} \rightarrow \Delta^{n} \otimes \Delta^{\varphi}\right\|_{P}\right) \simeq\left\|\partial\left(\Delta^{n}\right) \otimes \Delta^{\alpha \circ \varphi} \rightarrow \Delta^{n} \otimes \Delta^{\alpha \circ \varphi}\right\|_{Q}
$$

If $\Delta^{\alpha \circ \varphi}$ is a non-degenerate simplex of $N(Q),(5)$ is a generating cofibration of $\operatorname{Top}_{Q}$, if not, it is the retract of a cofibration by Lemma 3.3. The same argument shows that $\alpha_{*}$ preserves trivial cofibrations, and so $\alpha_{*}$ is a left Quillen functor.

If $\alpha$ is an isomorphism, $\alpha_{*}$ and $\alpha^{*}$ are inverse equivalences of categories. Furthermore, $\alpha^{*}$ and $\alpha_{*}$ map the set of generating (trivial) cofibrations of $\operatorname{Top}_{P}$ and $\operatorname{Top}_{Q}$ to each other (up to isomorphism), which means that $\left(\alpha_{*}, \alpha^{*}\right)$ is a Quillen-equivalence. Conversely, suppose $p_{0} \neq p_{1}$ satisfy $\alpha\left(p_{0}\right)=$ $\alpha\left(p_{1}\right)=q \in Q$. Consider the stratified space $X=\left(\{*\}, P, * \mapsto p_{0}\right)$. It is cofibrant, and one has $\alpha^{*} \alpha_{*}(X)=\left(\alpha^{-1}\{q\}, P, \alpha^{-1}(q) \subset P\right)$. In particular, $s \pi_{0}\left(\alpha^{*} \alpha_{*}(X)\right)\left(\left\{p_{1}\right\}\right) \neq \emptyset=s \pi_{0}(X)$, and so $X \rightarrow \alpha^{*} \alpha_{*}(X)$ is not a weak-equivalence. Next assume that there exist $q \in Q$ not in the image of $\alpha$, and let $Y=(\{*\}, Q, * \mapsto q)$. Then, $\alpha_{*} \alpha^{*}(Y)$ is empty. In particular, $\alpha_{*} \alpha^{*}(Y) \rightarrow Y$ is not a weak-equivalence. Finally, assume that $\alpha$ is a bijection and that there exist $p_{0}, p_{1} \in P$ such that $p_{0} \nless p_{1}$ but $\alpha\left(p_{0}\right)=q_{0}<q_{1}=\alpha\left(p_{1}\right)$. Consider $X=\left(\left\{p_{0}, p_{1}\right\}, P,\left\{p_{0}, p_{1}\right\} \hookrightarrow P\right)$ in $\operatorname{Top}_{P}$ and $Y=\left(\left\|\Delta^{\varphi}\right\|_{Q}, Q, \varphi_{Q}\right)$ in $\operatorname{Top}_{Q}$, where $\Delta^{\varphi}=\left\{q_{0}<q_{1}\right\}$ is a non-degenerate simplex of $N(Q)$. Then, consider the map $i: \alpha_{*}(X) \rightarrow Y$ which corresponds to the inclusion $\left\|\partial\left(\Delta^{\varphi}\right)\right\|_{Q} \rightarrow\left\|\Delta^{\varphi}\right\|_{Q}$. Its adjoint is a weak-equivalence, since $D_{P}(X)\left(p_{0}\right) \sim\{*\} \sim D_{P}\left(\alpha^{*}(Y)\right)\left(p_{0}\right)$ and $D_{P}(X)\left(p_{1}\right) \sim\{*\} \sim$ $D_{P}\left(\alpha^{*}(Y)\right)$. But $i$ is not a weak-equivalence since $D_{Q}\left(\alpha_{*}(X)\right)\left(\Delta^{\varphi}\right)=\emptyset$ but $D_{Q}(Y)\left(\Delta^{\varphi}\right) \neq \emptyset$.

Lemma 3.3. Let $\varphi: \Delta^{n} \rightarrow N(P)$ be a not-necessarily injective simplicial map. Then, there exists an injective map $\bar{\varphi}: \Delta^{k} \rightarrow N(P)$ and a topological space $K$ such that $\left\|\Delta^{\varphi}\right\|_{P}$ is a retract of $\left\|\Delta^{\bar{\varphi}}\right\|_{P} \otimes K$

Proof. Let $\bar{\varphi}: \Delta^{k} \rightarrow N(P)$ be the inclusion $\operatorname{Im}(\varphi) \rightarrow N(P)$. Enumerate the vertices of $\Delta^{k} \in$ $N(P)$ as $\left\{p_{0}<\cdots<p_{k}\right\}$. Enumerate the vertices of $\Delta^{n}$ as $\left\{e_{0}^{p_{0}}, e_{1}^{p_{0}}, \ldots, e_{m_{0}}^{p_{0}}, e_{0}^{p_{1}}, \ldots, e_{m_{k}}^{p_{k}}\right\}$, where 
$\varphi\left(e_{i}^{p_{j}}\right)=p_{j}$. Consider the map defined on vertices as

$$
\begin{aligned}
f: \Delta^{k} \times \Delta^{n} & \rightarrow \Delta^{n} \\
\left(p_{l}, e_{i}^{p_{j}}\right) & \mapsto \begin{cases}e_{i}^{p_{j}} & \text { if } p_{j}=p_{l} \\
e_{0}^{p_{l}} & \text { if } p_{j} \neq p_{l}\end{cases}
\end{aligned}
$$

It extends to a map of simplicial sets. We then have the following retract

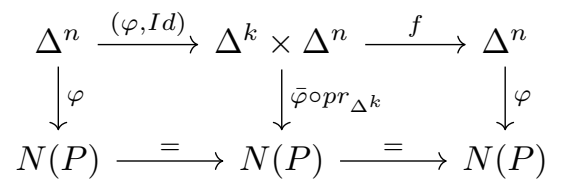

Taking the realization gives the desired retract.

\subsection{The model category Strat.}

Definition 3.4. Let $f:\left(X, P, \varphi_{X}\right) \rightarrow\left(Y, Q, \varphi_{Y}\right)$ be a stratified map, such that $\widehat{f}: P \rightarrow Q$ is an isomorphism of posets. We will also write $\widehat{f}$ for the corresponding isomorphism $\widehat{f}: N(P) \rightarrow N(Q)$. Define the functor $s \pi_{0}\left(Y, Q, \varphi_{Y}\right) \circ \widehat{f}$ as follows

$$
\begin{aligned}
s \pi_{0}\left(Y, Q, \varphi_{Y}\right) \circ \widehat{f}: R(P)^{o p} & \rightarrow \text { Set } \\
\Delta^{\psi} & \mapsto s \pi_{0}\left(Y, Q, \varphi_{Y}\right)\left(\Delta^{\widehat{f} \circ \psi}\right)
\end{aligned}
$$

Let $\phi:\left\|\Delta^{\varphi}\right\|_{P} \rightarrow\left(X, P, \varphi_{X}\right)$ be a pointing, and $n \geq 1$ an integer. Define the pointing of $\left(Y, Q, \varphi_{Y}\right)$, $f \circ \phi$ as

$$
f \circ \phi:\left\|\Delta^{\widehat{f} \circ \varphi}\right\|_{Q} \rightarrow\left(Y, Q, \varphi_{Y}\right)
$$

Then, define the functor $s \pi_{n}\left(\left(Y, Q, \varphi_{Y}\right), f \circ \phi\right) \circ \widehat{f}$ as follows

$$
\begin{aligned}
s \pi_{n}\left(\left(Y, Q, \varphi_{Y}\right), f \circ \phi\right) \circ \widehat{f}: R(\operatorname{Im}(\varphi))^{o p} & \rightarrow \text { Set } \\
\Delta^{\psi} & \mapsto s \pi_{n}\left(\left(Y, Q, \varphi_{Y}\right), f \circ \phi\right)\left(\Delta^{\widehat{f} \circ \psi}\right)
\end{aligned}
$$

Proposition 3.5. Let $f:\left(X, P, \varphi_{X}\right) \rightarrow\left(Y, Q, \varphi_{Y}\right)$ be a stratified map, such that $\widehat{f}: P \rightarrow Q$ is an isomorphism of posets. Then, $f$ induces a morphism of functors

$$
s \pi_{0}(f): s \pi_{0}\left(X, P, \varphi_{X}\right) \rightarrow s \pi_{0}\left(Y, Q, \varphi_{Y}\right) \circ \widehat{f}
$$

Furthermore, for all pointing of $\left(X, P, \varphi_{X}\right), \phi$, and all $n \geq 1, f$ induces morphisms of functors

$$
s \pi_{n}(f): s \pi_{n}\left(\left(X, P, \varphi_{X}\right), \phi\right) \rightarrow s \pi_{n}\left(\left(Y, Q, \varphi_{Y}\right), f \circ \phi\right) \circ \widehat{f}
$$

Proof. Define $s \pi_{n}(f)$ as follows. An element in $s \pi_{n}\left(\left(X, P, \varphi_{X}\right), \phi\right)\left(\Delta^{\varphi}\right)$ is an element in

$$
\pi_{n}\left(\operatorname{Sing}\left(\mathcal{C}_{P}^{0}\left(\left\|\Delta^{\varphi}\right\|_{P},\left(X, P, \varphi_{X}\right)\right)\right), \phi_{\|\|^{\varphi} \|_{P}}\right)
$$

Using the isomorphism $\alpha: P \rightarrow Q$ this is in bijection with

$$
\pi_{n}\left(\operatorname{Sing}\left(\mathcal{C}_{Q}^{0}\left(\left\|\Delta^{\alpha \circ \varphi}\right\|_{Q},\left(X, Q, \alpha \circ \varphi_{X}\right)\right)\right), \phi_{\|\| \Delta^{\alpha \circ \varphi} \|_{Q}}\right)
$$

the map $f$ induces a map $f_{\triangleright}:\left(X, P, \alpha \circ \varphi_{X}\right) \rightarrow\left(Y, \varphi_{Y}, Q\right)$ in $\operatorname{Top}_{Q}$ (see Proposition 3.7) which in turns sends an element of (7) to an element in

$$
\pi_{n}\left(\operatorname{Sing}\left(\mathcal{C}_{Q}^{0}\left(\left\|\Delta^{\alpha \circ \varphi}\right\|_{Q},\left(Y, Q, \varphi_{Y}\right)\right)\right), f \circ \phi_{\|\| \Delta^{\alpha \circ \varphi} \|_{Q}}\right)
$$


The composition gives us a map

$$
\begin{gathered}
s \pi_{n}\left(\left(X, P, \varphi_{X}\right), \phi\right)\left(\Delta^{\varphi}\right) \\
\simeq \downarrow \\
\qquad \pi_{n}\left(\left(X, Q, \alpha \circ \varphi_{X}\right), \phi\right)\left(\Delta^{\alpha \circ \varphi}\right) \underset{s \pi_{n}\left(f_{\triangleright}\right)}{\longrightarrow} s \pi_{n}\left(\left(Y, Q, \varphi_{Y}\right), f \circ \phi\right)\left(\Delta^{\alpha \circ \varphi}\right)
\end{gathered}
$$

Which is exactly the map (6) evaluated at $\Delta^{\varphi}$.

The goal of this section is to prove the following theorem

Theorem 3.6. There exists a cofibrantly generated model structure on Strat such that a stratified map $f:\left(X, P, \varphi_{X}\right) \rightarrow\left(Y, Q, \varphi_{Y}\right)$ is a weak-equivalence if and only if $\widehat{f}: P \rightarrow Q$ is an isomorphism of poset, and if the map

$$
s \pi_{0}(f): s \pi_{0}\left(X, P, \varphi_{X}\right) \rightarrow s \pi_{0}\left(Y, Q, \varphi_{Y}\right) \circ \widehat{f}
$$

is an isomorphism, and if for all pointings of $X, \phi$, and all $n \geq 1$, the map

$$
s \pi_{n}(f): s \pi_{n}\left(\left(X, P, \varphi_{X}\right), \phi\right) \rightarrow s \pi_{n}\left(\left(Y, Q, \varphi_{Y}\right), f \circ \phi\right) \circ \widehat{f}
$$

is an isomorphism. The generating set of cofibration and trivial cofibrations are the following

- $I=\left\{S^{n-1} \times\left\|\Delta^{\varphi}\right\|_{\mathbb{N}} \rightarrow D^{n} \times\left\|\Delta^{\varphi}\right\|_{\mathbb{N}} \mid n \geq 0, \varphi: \Delta^{k} \rightarrow N(\mathbb{N})\right\}$

- $J=\left\{D^{n} \times\left\|\Delta^{\varphi}\right\|_{\mathbb{N}} \rightarrow D^{n} \times[0,1] \times\left\|\Delta^{\varphi}\right\|_{\mathbb{N}} \mid n \geq 0, \varphi: \Delta^{k} \rightarrow N(\mathbb{N})\right\}$

In order to prove Theorem 3.6 , we will need the following :

Proposition 3.7. Let $f:\left(X, P, \varphi_{X}\right) \rightarrow\left(Y, Q, \varphi_{Y}\right)$ be a stratified map, and write $\alpha=\widehat{f}: P \rightarrow Q$. The stratified map $f$ admits the following two factorisations :

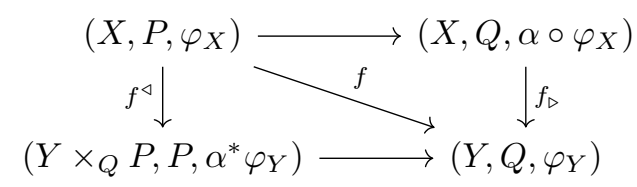

where $f^{\triangleleft}$ is a map in $\operatorname{Top}_{P}$ and $f_{\triangleright}$ is a map in $\operatorname{Top}_{Q}$.

Proof. The filtered map $f_{\triangleright}$ is simply $f: X \rightarrow Y$ together with $I d_{Q}$, and we clearly have $(f, \alpha)=$ $\left(I d_{X}, \alpha\right) \circ\left(f, I d_{Q}\right)$. We obtain the other half of the square by adjunction.

We can now define the following classes of maps.

Definition 3.8. A stratified map $f:\left(X, P, \varphi_{X}\right) \rightarrow\left(Y, Q, \varphi_{Y}\right)$ is :

- a stratified cofibration if $f_{\triangleright}$ is a cofibration in $\operatorname{Top}_{Q}$.

- a stratified fibration if $f^{\triangleleft}$ is a fibration in $\operatorname{Top}_{P}$.

- a stratified trivial cofibration if $f_{\triangleright}$ is a trivial cofibration in $\operatorname{Top}_{Q}$.

- a stratified trivial fibration if $f^{\triangleleft}$ is a trivial fibration in $\operatorname{Top}_{P}$.

Proof of Theorem 3.6. Consider the functor

$$
\begin{aligned}
F: \text { Strat } & \rightarrow \text { Poset } \\
\left(X, P, \varphi_{X}\right) & \mapsto P
\end{aligned}
$$

where Poset is the category of posets and order preserving maps. It is a Grothendieck bifibration [CM17, Definition 2.1]. Consider the trivial model structure on Poset, where weak-equivalences are 
the isomorphisms, and all maps are cofibrations and fibrations. By proposition 3.2 hypothesis $(Q)$ of [CM17, Theorem 4.2] is verified, and the other hypothesis follow easily from the fact that Poset is taken with the trivial model structure. Applying [CM17, Theorem 4.2] to $F$ gives a model structure on Strat with the classes of (trivial) cofibrations and fibrations of definition 3.8 Let us show that it coincides with the model structure described in Theorem 3.6. Let $f:\left(X, P, \varphi_{X}\right) \rightarrow\left(Y, Q, \varphi_{Y}\right)$ be a stratified map, and factor it as a trivial cofibration followed by a fibration.

$$
\left(X, P, \varphi_{X}\right) \stackrel{j}{\longrightarrow}\left(Z, R, \varphi_{Z}\right) \stackrel{p}{\longrightarrow}\left(Y, Q, \varphi_{Y}\right)
$$

Write $\widehat{j}=\beta$ and $\widehat{p}=\gamma$, and consider the following commutative diagram :

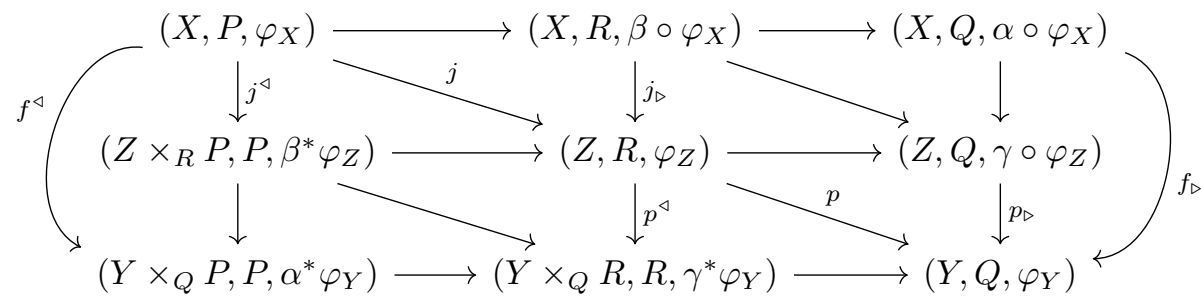

$f$ is a weak-equivalence if and only if $p$ is a trivial fibration. The latter is true if and only if $\gamma$ is an isomorphism and if $p^{\triangleleft}$ is a trivial fibration. If $\gamma$ is an isomorphism, all rows are weak-equivalences. By repeated applications of the two out of three property, we get that $f$ is a weak-equivalence if and only if all rows and columns are weak-equivalences. In particular, $f$ is a weak-equivalenc if and only if $f^{\triangleleft}$ is a weak-equivalence in $\operatorname{Top}_{P}$. Lemma 3.9 then gives the desired characterization of weak-equivalences.

It remains to show that $I$ and $J$ are sets of generating (trivial) cofibrations. Let $f:\left(X, P, \varphi_{X}\right) \rightarrow$ $\left(Y, Q, \varphi_{Y}\right)$ be a stratified map, and consider the following lifting problem

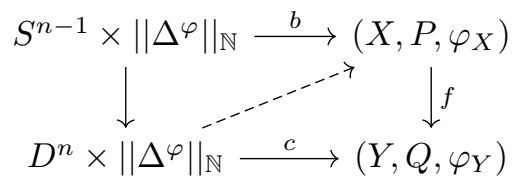

Write $\widehat{f}=\alpha, \widehat{b}=\beta: \mathbb{N} \rightarrow P$ and $\widehat{c}=\gamma: \mathbb{N} \rightarrow Q$. The commutativity of the diagram (8) guarantees that $\alpha \circ \beta=\gamma$. Factoring $c$ as follows

$$
D^{n} \times\left\|\Delta^{\varphi}\right\|_{\mathbb{N}} \stackrel{d}{\longrightarrow} D^{n} \times\left\|\Delta^{\alpha \circ \varphi}\right\|_{P} \stackrel{e}{\longrightarrow}\left(Y, Q, \varphi_{Y}\right)
$$

where $\widehat{d}=\beta$ and $\widehat{e}=\alpha$. We can now use adjunction $\left(\alpha_{*}, \alpha^{*}\right)$, to get a lifting problem in Top ${ }_{P}$, equivalent to (8).

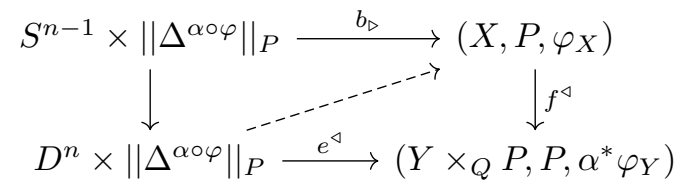

The maps $S^{n-1} \times\left\|\Delta^{\alpha \circ \varphi}\right\|_{P} \rightarrow D^{n} \times\left\|\Delta^{\alpha \circ \varphi}\right\|_{P}$ are not necessarily generating cofibration since $\alpha \circ \varphi: \Delta^{k} \rightarrow N(P)$ might not be injective, but by lemma $3.3 f^{\triangleleft}$ is a trivial fibration of $\operatorname{Top}_{P}$ if and only if it has the right lifting property against all maps of this form. In particular, $f$ is a trivial 
fibration if and only if it has the right lifting property against all maps of $I$. The proofs works similarly for the set of generating trivial cofibrations.

Lemma 3.9. Let $f:\left(X, P, \varphi_{X}\right) \rightarrow\left(Y, Q, \varphi_{Y}\right)$ be a stratified map such that $\widehat{f}=\alpha: P \rightarrow Q$ is an isomorphism of posets. Then $f^{\triangleleft}:\left(X, P, \varphi_{X}\right) \rightarrow\left(Y \times_{Q} P, P, \alpha^{*} \varphi_{Y}\right)$ is a weak-equivalence in Top $_{P}$ if and only if $f$ induces isomorphisms

$$
s \pi_{0}(f): s \pi_{0}\left(X, P, \varphi_{X}\right) \rightarrow s \pi_{0}\left(Y, Q, \varphi_{Y}\right) \circ \widehat{f}
$$

and,

$$
s \pi_{n}(f): s \pi_{n}\left(\left(X, P, \varphi_{X}\right), \phi\right) \rightarrow s \pi_{n}\left(\left(Y, Q, \varphi_{Y}\right), f \circ \phi\right) \circ \widehat{f}
$$

for all pointing of $\left(X, P, \varphi_{X}\right), \phi$, and all $n \geq 1$.

Proof. The map induced by $f$ on stratified homotopy groups factors in two ways as follows

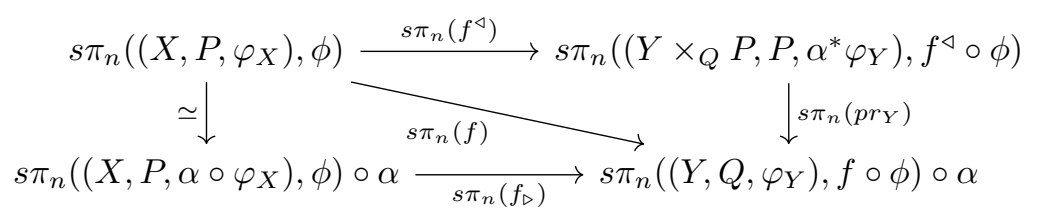

Now, $f^{\triangleleft}$ is a weak-equivalence if and only if $s \pi_{n}\left(f^{\triangleleft}\right)$ is an isomorphism for all $n$ and $\phi$. But the latter is true if and only if $s \pi_{n}(f)$ is an isomorphism for all $n$ and $\phi$, since $s \pi_{n}\left(p r_{Y}\right)$ is an isomorphism.

Remark 3.10. The proof of Theorem 3.6- and of Proposition 3.2 on which it depends - generalizes without difficulty to the category StrStrat of strongly stratified spaces or even to a category of strongly stratified simplicial sets. In particular, there exists a cofibrantly generated model category of strongly stratified spaces.

\section{ApPEndix A. A PROPERTY OF SOME COLIMITS OVER PARTICULAR POSETS}

Definition A.1. Let $\mathcal{D}$ be a poset and $G: \mathcal{D} \rightarrow$ Set be a functor. We say that $\mathcal{D}$ is almost filtered with respect to $G$ if,

(1) for all $d \in \mathcal{D}$ and all $d_{1}, d_{2}, d_{3} \in \mathcal{D}$ such that $d_{1} \leq d, d_{2}$, and $d_{3} \leq d_{2}, d$, if there exist $x, y \in G(d)$ and $x_{i} \in G\left(d_{i}\right), i \in\{1,2,3\}$, such that $G\left(d_{1} \rightarrow d\right)\left(x_{1}\right)=x, G\left(d_{1} \rightarrow d_{2}\right)\left(x_{1}\right)=$ $x_{2}, G\left(d_{3} \rightarrow d_{2}\right)\left(x_{3}\right)=x_{2}$ and $G\left(d_{3} \rightarrow d\right)\left(x_{3}\right)=y$, then there exists $e \in \mathcal{D}$ such that the following relations are satisfied,

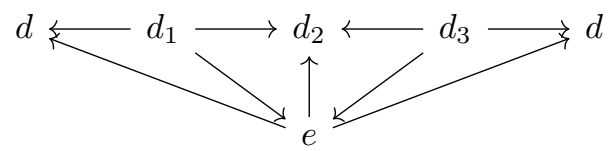

(2) - for all $n \geq 2$, and all $(2 n+3)$-tuple in $\mathcal{D},\left(d_{0}, \ldots, d_{2 n+2}\right)$, with $d_{0}=d_{2 n+2}=d$, and $d_{2 k+1} \leq d_{2 k}, d_{2 k+2}$, for all $0 \leq k \leq n$,

- for all $(2 n+3)$-tuple $\left(x_{0}, \ldots, x_{2 n+2}\right)$, such that $x_{i} \in G\left(d_{i}\right)$, for $0 \leq i \leq 2 n+2$, and $G\left(d_{2 k+1} \rightarrow d_{2 k}\right)\left(x_{2 k+1}\right)=x_{2 k}$ and $G\left(d_{2 k+1} \rightarrow d_{2 k+2}\right)\left(x_{2 k+1}\right)=x_{2 k+2}$ for all $0 \leq k \leq n$.

there exists : 
- a $(2 n+3)$-tuple of elements in $\mathcal{D},\left(d_{0}^{\prime}, \ldots, d_{2 n+2}^{\prime}\right)$ and $e$ an element in $\mathcal{D}$, such that $d_{0}^{\prime}=d_{2 n+1}^{\prime}=d$, and such that the relation depicted in the following diagram are satisfied

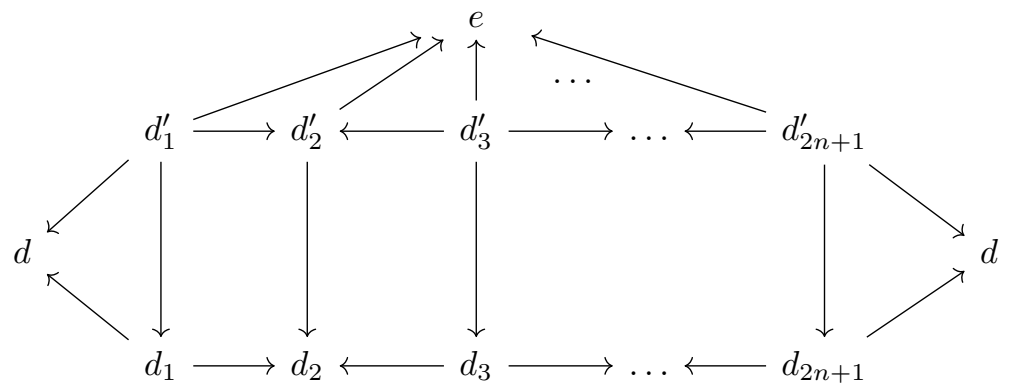

- a $(2 n+3)$-tuple $\left(x_{0}^{\prime}, \ldots, x_{2 n+2}^{\prime}\right)$ such that $x_{i}^{\prime} \in G\left(d_{i}^{\prime}\right)$ for all $0 \leq i \leq 2 n+2$ and $G\left(d_{2 k+1}^{\prime} \rightarrow d_{2 k}^{\prime}\right)\left(x_{2 k+1}^{\prime}\right)=x_{2 k}^{\prime}$ and $G\left(d_{2 k+1}^{\prime} \rightarrow d_{2 k+2}^{\prime}\right)\left(x_{2 k+1}^{\prime}\right)=x_{2 k+2}^{\prime}$ for all $0 \leq k \leq$ $n$.

such that $G\left(d_{i}^{\prime} \rightarrow d_{i}\right)\left(x_{i}^{\prime}\right)=x_{i}$ for all $0 \leq i \leq 2 n+2$.

Proposition A.2. Let $\mathcal{D}$ be a poset, and $G: \mathcal{D} \rightarrow$ Set a functor sending morphisms of $\mathcal{D}$ to monomorphisms in Set. If $\mathcal{D}$ is almost filtered with respect to $G$, then for any $d \in \mathcal{D}$, the map

$$
G(d) \rightarrow \operatorname{colim}_{d \in \mathcal{D}}(G)
$$

is a monomorphism.

Proof. Recall the following

$$
\underset{\mathcal{D}}{\operatorname{colim}} G=\left(\coprod_{d \in \mathcal{D}} G(d)\right) / \sim
$$

Where $\sim$ is the equivalence relation generated by $x \sim y$ if $x \in G(c), y \in G(d)$ and $G(c \rightarrow d)(x)=y$. It suffices to prove that if $d \in \mathcal{D}$ and $x, y$ are two elements of $G(d)$, then

$$
x \sim y \Leftrightarrow x=y .
$$

Assume that $x \sim y$. Then, there exists a zigzag of maps in $\mathcal{D}$, realizing this equivalence, of one of the following three forms

$$
\begin{gathered}
d \longleftarrow d_{1} \longrightarrow \ldots \longleftarrow d_{2 n+1} \longrightarrow d \\
d \longleftarrow d_{1} \longrightarrow d_{2} \longleftarrow \ldots \longrightarrow d_{2 n} \longleftarrow d \\
d \longrightarrow d_{1} \longleftarrow \ldots \longrightarrow d_{2 n+1} \longleftarrow d
\end{gathered}
$$

Composing the first map of (13) with $d \rightarrow d_{2 n}$, and omitting the last gives a zigzag of shape (12) between $G\left(d \rightarrow d_{2 n}\right)(x)$ and $G\left(d \rightarrow d_{2 n}\right)(y)$. Since $G\left(d \rightarrow d_{2 n}\right)$ is a monomorphism, showing that $x=y$ is equivalent to showing that the two former elements of $G\left(d_{2 n}\right)$ are equal. Similarly, omitting the first map in (14) and adding the map $d \rightarrow d_{1}$ at the end gives a zigzag of shape (12) between $G\left(d \rightarrow d_{1}\right)(x)$ and $G\left(d \rightarrow d_{1}\right)(y)$. Since $G\left(d \rightarrow d_{1}\right)$ is a monomorphism by assumption, those elements of $G\left(d_{1}\right)$ are equal if and only if $x=y$. In particular, it is enough to show that the existence of a zigzag of shape (12) realizing an equivalence $x \sim y$, implies that $x=y$. Fix such a 
zigzag, and elements $x_{i} \in G\left(d_{i}\right)$, for all $0 \leq i \leq 2 n+2$ (take $x_{0}=x$, and $x_{2 n+2}=y$ ), such that $G\left(d_{2 k+1} \rightarrow d_{2 k}\right)\left(x_{2 k+1}\right)=x_{2 k}$ and $G\left(d_{2 k+1} \rightarrow d_{2 k+2}\right)\left(x_{2 k+1}\right)=x_{2 k+2}$.

Assume that $n \geq 2$. Since $\mathcal{D}$ is almost filtered with respect to $G$, there exist $e \in \mathcal{D}$ and a tuple, $\left(d_{1}^{\prime}, \ldots, d_{2 n+1}^{\prime}\right)$ such that the relations of (10) hold. Furthermore, there exists $\left(x_{1}^{\prime}, \ldots, x_{2 n+1}^{\prime}\right)$ such that the relation of Definition A.1 are satisfied. The commutativity of (10) guarantees that $x_{e}=G\left(d_{i}^{\prime} \rightarrow e\right)\left(x_{i}^{\prime}\right)$ is well defined and independant of the choice of $i$. In particular, we have a zigzag of the form

$$
d \longleftarrow d_{1}^{\prime} \longrightarrow e \longleftarrow d_{2 n+1}^{\prime} \longrightarrow d
$$

realizing the equivalence $x \sim y$. This is exactly a zigzag of shape (12), with $n=1$.

Assume that $n=1$. Since $\mathcal{D}$ is almost filtered with respect to $G$, there exists $e \in \mathcal{D}$ such that there is a diagram of shape (9). Taking $x_{e}=G\left(d_{1} \rightarrow e\right)\left(x_{1}\right)=G\left(d_{3} \rightarrow e\right) \in G(e)$, we get a zigzag of shape (12), with $n=0$.

Assume that $n=0$. This means that there exists $d_{1} \in \mathcal{D}$, and $x_{1}$ such that $G\left(d_{1} \rightarrow d\right)\left(x_{1}\right)=x$ and $G\left(d_{1} \rightarrow d\right)\left(x_{1}\right)=y$. Clearly, this implies that $x=y$.

Remark A.3. At fist glance, one might think that the hypothesis in Proposition $A .2$ that $\mathcal{D}$ is almost filtered with respect to $G$ is unnecessary, and that the conclusion should hold for any functor $G: \mathcal{D} \rightarrow$ Set sending morphisms of $\mathcal{D}$ to monomorphisms, as long as $\mathcal{D}$ is a poset. But this is not true. Consider the poset $\mathcal{D}$ with four objects $a, b, c$ and $d$ and relations $a \rightarrow c, a \rightarrow d$, $b \rightarrow c, b \rightarrow d$, and the functor $G: \mathcal{D} \rightarrow$ Set defined by $G(a)=G(b)=G(c)=G(d)=\{0,1\}$, and $G(a \rightarrow c)=G(a \rightarrow d)=G(b \rightarrow c)=I d$, and $G(b \rightarrow d)=1-I d$. Then, colim ${ }_{\mathcal{D}} G=\{*\}$.

Remark A.4. One can see any (filtered) simplicial set as a set by considering its set of simplices. Indeed, if $\left(X, \varphi_{X}\right)$ is a filtered simplicial set, and $\Delta^{\varphi} \in N(P)$ is a simplex of $N(P)$, write $X_{\Delta \varphi}$ for the set of $\Delta^{\varphi}$-simplices of $X$ (equivalently, for the set of filtered maps $\operatorname{Hom}_{\mathrm{sSet}}\left(\Delta^{\varphi},\left(X, \varphi_{X}\right)\right.$ ). The set of simplices of $X$ is then the disjoint union $U(X)=\underset{\Delta^{\varphi} \in \Delta(P)}{\coprod} X_{\Delta^{\varphi}}$. Notice that a map of (filtered) simplicial sets $\left(X, \varphi_{X}\right) \rightarrow\left(Y, \varphi_{Y}\right)$ is a monomorphism if and only if the corresponding map of sets $U(X) \rightarrow U(Y)$ is a monomorphism. In the same way, one can see any functor with values in the category of (filtered) simplicial sets, as a functor with values in Set by composing it with the forgetful functor $U$. Further, let $\mathcal{C}$ be a small category and $F: \mathcal{C} \rightarrow \operatorname{sSet}_{P}$ be a functor, for all $\Delta^{\varphi} \in \Delta(P)$, one defines the functor $F_{\Delta^{\varphi}}: \mathcal{C} \rightarrow \operatorname{sSet}_{P}$ by taking $F_{\Delta^{\varphi}}(c)=F(c)_{\Delta \varphi}$ for all $c \in \mathcal{C}$. One then has:

$$
\left(\operatorname{colim}_{c \in \mathcal{C}} F(c)\right)_{\Delta^{\varphi}}=\operatorname{colim}_{c \in \mathcal{C}}\left(F_{\Delta \varphi}(c)\right)
$$

Equivalently, one says that the limits and colimits in $\operatorname{sSet}_{P}$ are computed degree-wise. Now since $U \circ F$ is a colimit of the $F_{\Delta \varphi}$, and since colimits commute with each-other, one has :

$$
\underset{\mathcal{C}}{\operatorname{colim}}(U \circ F)=U(\underset{\mathcal{C}}{\operatorname{colim}} F) .
$$

In particular, for the matter of studying colimits of a functor with values in $\operatorname{sSet}_{P}$, one can see it as a functor with values in Set by composing with the forgetful functor $U$. Furthermore, since $U$ reflects monomorphisms, if Proposition A.2 applies to the functor with values in Set, it will also apply to the original functor.

Lemma A.5. Let $F$ be an object in $\operatorname{Diag}_{P}$ for some poset $P$. Then $\mathcal{C}$ is almost filtered with respect to $F \otimes R(P)$, seen as a functor to Set. (See Definition 2.5.) 
Proof. Consider a diagram in $\mathcal{C}$ of shape

$$
\left(\Delta^{\varphi}, \Delta^{\psi}\right) \longleftarrow\left(\Delta^{\varphi_{1}}, \Delta^{\psi_{1}}\right) \rightarrow\left(\Delta^{\varphi_{2}}, \Delta^{\psi_{2}}\right) \longleftarrow\left(\Delta^{\varphi_{3}}, \Delta^{\psi_{3}}\right) \rightarrow\left(\Delta^{\varphi}, \Delta^{\psi}\right)
$$

And simplices $\sigma, \tau \in F\left(\Delta^{\varphi}\right) \otimes \Delta^{\psi}$, and $\sigma_{i} \in F\left(\Delta^{\varphi_{i}}\right) \otimes \Delta^{\psi_{i}}$, for $i \in\{1,2,3\}$, related to each other as in Definition A.1. Write each of the $\sigma_{i}$ as a pair $\left(\sigma_{i}^{F}, \sigma_{i}^{P}\right)$, where $\sigma_{i}^{F} \in F\left(\Delta^{\varphi_{i}}\right)$ and $\sigma_{i}^{P} \in \Delta^{\psi_{i}}$. By construction of $F \otimes R(P)$, we have $\sigma^{P}=\sigma_{i}^{P}=\tau^{P}$, for all $1 \leq i \leq 3$, and in particular, $\Delta^{\psi} \cap \Delta^{\psi_{2}}$ is not empty, write $\Delta^{\psi^{\prime}}$ for this intersection. Furthermore, since $\Delta^{\varphi_{1}}$ contains both $\Delta^{\varphi}$ and $\Delta^{\varphi_{2}}$, there exists a smallest simplex of $N(P)$ - write it $\Delta^{\varphi^{\prime}}$ - containing $\Delta^{\varphi} \cup \Delta^{\varphi_{2}}$. We then get the following factorization

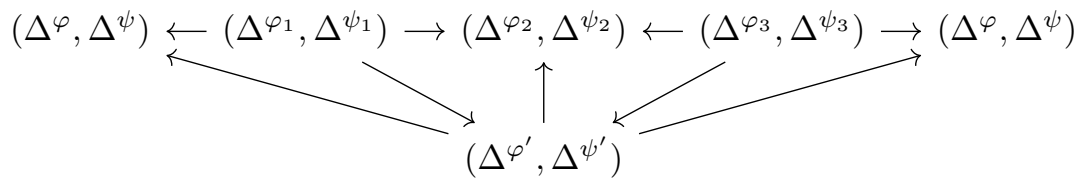

Next, assume that we are given a family of $\left(\Delta^{\varphi_{i}}, \Delta^{\psi_{i}}\right)$, together with $\sigma_{i} \in F\left(\Delta^{\varphi_{i}}\right) \otimes \Delta^{\psi_{i}}, 0 \leq i \leq$ $2 n+2$, as in Definition A.1. Then as earlier, write $\sigma_{i}=\left(\sigma_{i}^{F}, \sigma_{i}^{P}\right)$. One must have $\sigma_{i}^{P}=\sigma_{j}^{\bar{P}}$, for all $0 \leq i, j \leq 2 n+2$, and so, the intersection $\cap_{i} \Delta^{\psi_{i}}$ must be non-empty, since it contains $\sigma_{i}^{P}$, for all $i$, write it $\Delta^{\psi^{\prime}}$. Then, the intersection $\cap_{i} \Delta^{\varphi_{i}}$ is also non-empty since it contains $\Delta^{\psi^{\prime}}$, write it $\Delta^{\varphi^{\prime}}$. To obtain a diagram of shape (10), pick $d_{i}^{\prime}=\left(\Delta^{\varphi_{i}}, \Delta^{\psi^{\prime}}\right)$, and $e=\left(\Delta^{\varphi^{\prime}}, \Delta^{\psi^{\prime}}\right)$. As mentioned earlier, the $\sigma_{i}^{P}$ can already be seen as elements of $\Delta^{\psi^{\prime}}$, so one can pick $x_{i}^{\prime}=x_{i}$.

\section{REFERENCES}

$\left[\mathrm{B}^{+} 08\right] \quad \mathrm{A}$. Borel et al. Intersection cohomology. Modern Birkhäuser Classics. Birkhäuser Boston, Inc., Boston, MA, 2008. Notes on the seminar held at the University of Bern, Bern, 1983, Reprint of the 1984 edition.

[Ban10] Markus Banagl. Rational generalized intersection homology theories. Homology Homotopy Appl., 12(1):157-185, 2010.

[BGH18] Clark Barwick, Saul Glasman, and Peter Haine. Exodromy. arXiv e-prints, page arXiv:1807.03281, Jul 2018.

[CM17] Pierre Cagne and Paul-André Melliès. On bifibrations of model categories. arXiv e-prints, page arXiv:1709.10484, Sep 2017.

[CSAT18] David Chataur, Martintxo Saralegi-Aranguren, and Daniel Tanré. Intersection cohomology, simplicial blow-up and rational homotopy. Mem. Amer. Math. Soc., 254(1214):viii+108, 2018.

[Dou18] S. Douteau. A Simplicial Approach to Stratified Homotopy Theory. ArXiv e-prints, January 2018.

[Dug03] Daniel Dugger. Notes on delta generated spaces. 2003.

[FM13] Greg Friedman and James E. McClure. Cup and cap products in intersection (co)homology. Adv. Math., 240:383-426, 2013.

[Fri] Greg Friedman. Singular Intersection Homology. http://faculty.tcu.edu/gfriedman/IHbook.pdf

[GM80] Mark Goresky and Robert MacPherson. Intersection homology theory. Topology, 19(2):135-162, 1980.

[GM83] Mark Goresky and Robert MacPherson. Intersection homology. II. Invent. Math., 72(1):77-129, 1983.

[Hai18] Peter J. Haine. A model for the $\infty$-category of stratified spaces. arXiv e-prints, page arXiv:1811.01119, Nov 2018.

[Hen] A. Henriques. A model category for stratified spaces. Preprint available at http://andreghenriques.com/PDF/Model_Cat_Stratified_spaces.pdf

[Hir03] Philip S. Hirschhorn. Model categories and their localizations, volume 99 of Mathematical Surveys and Monographs. American Mathematical Society, Providence, RI, 2003.

[HKRS17] Kathryn Hess, Magdalena Kedziorek, Emily Riehl, and Brooke Shipley. A necessary and sufficient condition for induced model structures. Journal of Topology, 10(2):324-369, 2017.

[Kin85] Henry C. King. Topological invariance of intersection homology without sheaves. Topology and its Applications, 20(2):149 - 160, 1985. 
[Lur] Jacob Lurie. Higher Algebra. http://www.math.harvard.edu/ lurie/papers/HA.pdf

[Mil57] John Milnor. The geometric realization of a semi-simplicial complex. Ann. of Math. (2), 65:357-362, 1957.

[Mil13] David A. Miller. Strongly stratified homotopy theory. Trans. Amer. Math. Soc., 365(9):4933-4962, 2013.

[NL19] Stephen Nand-Lal. A simplicial appraoch to stratified homotopy theory. PhD thesis, University of Liverpool, 2019.

[Qui88] Frank Quinn. Homotopically stratified sets. Journal of the American Mathematical Society, 1(2):441-499, 1988.

[Tho69] R. Thom. Ensembles et morphismes stratifiés. Bull. Amer. Math. Soc., 75:240-284, 1969.

[Tre09] David Treumann. Exit paths and constructible stacks. Compos. Math., 145(6):1504-1532, 2009.

[TT18] Dai Tamaki and Hiro Lee Tanaka. Stellar Stratifications on Classifying Spaces. arXiv e-prints, page arXiv:1804.11274, Apr 2018.

[Whi65] Hassler Whitney. Tangents to an analytic variety. Ann. of Math. (2), 81:496-549, 1965.

[Woo09] Jon Woolf. The fundamental category of a stratified space. J. Homotopy Relat. Struct., 4(1):359-387, 2009 . 